\title{
Illuminating dark photons with high-energy colliders
}

\author{
David Curtin, ${ }^{a}$ Rouven Essig, ${ }^{b}$ Stefania Gori ${ }^{c}$ and Jessie Shelton ${ }^{d}$ \\ ${ }^{a}$ Maryland Center for Fundamental Physics, University of Maryland, \\ College Park, MD 20742, U.S.A. \\ ${ }^{b}$ C.N. Yang Institute for Theoretical Physics, Stony Brook University, \\ Stony Brook, NY 11794, U.S.A. \\ ${ }^{c}$ Perimeter Institute for Theoretical Physics, \\ 31 Caroline St. N, Waterloo, Ontario, Canada \\ ${ }^{d}$ Dept. of Physics, University of Illinois at Urbana-Champaign, \\ 1110 West Green Street, Urbana, IL 61801, U.S.A. \\ E-mail: dcurtin1@umd.edu, rouven.essig@stonybrook.edu, \\ sgori@perimeterinstitute.ca, sheltonj@illinois.edu
}

ABSTRACT: High-energy colliders offer a unique sensitivity to dark photons, the mediators of a broken dark U(1) gauge theory that kinetically mixes with the Standard Model (SM) hypercharge. Dark photons can be detected in the exotic decay of the $125 \mathrm{GeV}$ Higgs boson, $h \rightarrow Z Z_{D} \rightarrow 4 \ell$, and in Drell-Yan events, $p p \rightarrow Z_{D} \rightarrow \ell \ell$. If the dark U(1) is broken by a hidden-sector Higgs mechanism, then mixing between the dark and SM Higgs bosons also allows the exotic decay $h \rightarrow Z_{D} Z_{D} \rightarrow 4 \ell$. We show that the $14 \mathrm{TeV} \mathrm{LHC}$ and a $100 \mathrm{TeV}$ proton-proton collider provide powerful probes of both exotic Higgs decay channels. In the case of kinetic mixing alone, direct Drell-Yan production offers the best sensitivity to $Z_{D}$, and can probe $\epsilon \gtrsim 9 \times 10^{-4}\left(4 \times 10^{-4}\right)$ at the HL-LHC (100 TeV pp collider). The exotic Higgs decay $h \rightarrow Z Z_{D}$ offers slightly weaker sensitivity, but both measurements are necessary to distinguish the kinetically mixed dark photon from other scenarios. If Higgs mixing is also present, then the decay $h \rightarrow Z_{D} Z_{D}$ can allow sensitivity to the $Z_{D}$ for $\epsilon \gtrsim 10^{-9}-10^{-6}\left(10^{-10}-10^{-7}\right)$ for the mass range $2 m_{\mu}<m_{Z_{D}}<m_{h} / 2$ by searching for displaced dark photon decays. We also compare the $Z_{D}$ sensitivity at $p p$ colliders to the indirect, but model-independent, sensitivity of global fits to electroweak precision observables. We perform a global electroweak fit of the dark photon model, substantially updating previous work in the literature. Electroweak precision measurements at LEP, Tevatron, and the LHC exclude $\epsilon$ as low as $3 \times 10^{-2}$. Sensitivity can be improved by up to a factor of $\sim 2$ with HL-LHC data, and an additional factor of $\sim 4$ with ILC/GigaZ data.

Keywords: Higgs Physics, Beyond Standard Model, Gauge Symmetry

ARXiv EPRINT: 1412.0018 


\section{Contents}

1 Introduction $\quad 1$

2 A kinetically mixed dark $\mathrm{U}(1) \quad 4$

2.1 The gauge sector 5

2.2 The Higgs sector 9

3 Constraining the hypercharge portal with electroweak precision observables

4 Constraining the hypercharge portal with $h \rightarrow Z Z_{D}$ decays $\quad 16$

5 Constraining the hypercharge portal with Drell-Yan $Z_{D}$ production 20

6 Constraining the Higgs and hypercharge portals with $h \rightarrow Z_{D} Z_{D}$ decays 22

6.1 Constraining the Higgs portal from prompt $Z_{D}$ decay 22

6.2 Constraints on kinetic mixing from displaced $Z_{D}$ decays 24

$\begin{array}{lll}7 & \text { Impact of future detector design } & 27\end{array}$

$\begin{array}{llr}8 & \text { Discussion and conclusions } & 29\end{array}$

A Tables of branching ratios and $Z_{D}$ full width 32

B $Z_{D}$ contributions to precision electroweak observables $\quad 34$

C MadGraph implementation of higgsed dark photon model 36

\section{Introduction}

The Large Hadron Collider (LHC) is dramatically increasing our understanding of physics at and beyond the electroweak scale. This major advance is not only due to the LHC's unprecedented center-of-mass energies, but also the large luminosity it is able to realize. This allows for the potential discovery of not just heavy states that carry Standard Model (SM) quantum numbers, but also light, weakly coupled states. Searches for such hiddensector degrees of freedom are an important component of the physics program at the LHC and future colliders, such as the envisioned $100 \mathrm{TeV}$ proton-proton collider [1-3] (see also [414]). Hidden sectors near the weak scale are motivated by naturalness [15-19], thermal dark matter [20-22], electroweak baryogenesis (see e.g. [23] for a recent review), but also represent a generic expectation for physics beyond the SM [24]. 
As a prototypical hidden sector, we consider the compelling possibility of a spontaneously broken "dark" $\mathrm{U}(1)_{D}$ gauge symmetry, mediated by a vector boson called the "dark photon", $Z_{D}$. The dark photon's only renormalizable interaction with the SM is through kinetic mixing with the hypercharge gauge boson [25-27]. In addition, if a dark Higgs mechanism is responsible for the spontaneous breaking of the $\mathrm{U}(1)_{D}$ gauge symmetry, the dark Higgs boson will in general have a renormalizable coupling to the $125 \mathrm{GeV}$ SM-like Higgs, resulting in a mixing between the two physical scalar states. The hidden sector's leading interactions with the SM may thus be through either the hypercharge portal, via the kinetic mixing coupling, which we denote as $\epsilon$, or through the Higgs portal, via the Higgs mixing, which we denote as $\kappa$. The impressive integrated luminosities achievable by the LHC and future hadron colliders make them powerful probes of the hidden sector through these two portals, while current and future electron-positron colliders can place interesting limits on kinetic mixing from precision electroweak tests (EWPTs), independently of the detailed spectrum of the hidden sector.

The dark photon mixes through the hypercharge portal with the SM photon and the $Z$ boson. If there are no hidden-sector states below the $Z_{D}$ mass, this mixing causes the dark photon to decay exclusively to SM particles, with sizable branching ratio to leptons. We will focus on the dark photon mass range $m_{Z_{D}}>2 m_{e} \sim 1 \mathrm{MeV}$, where the $Z_{D}$ can decay to SM fermions. ${ }^{1}$ There are many experimental probes of dark photons with a mass above $1 \mathrm{MeV}$ that decay directly to SM particles, including precision QED measurements, rare meson decays, supernova cooling, collider experiments, and beam dumps [31-66]. Most of the current effort in the search for dark photons above the $\mathrm{MeV}$-scale is devoted to $m_{Z_{D}} \lesssim 10 \mathrm{GeV}$, although see $[51,52,67-72]$ for recent discussions of exploring heavier $Z_{D}$. There is no compelling reason for not exploring the entire mass range that is experimentally accessible, since $m_{Z_{D}}$ is a free parameter of the theory. Dark photons with sub-GeV masses have received attention recently as they could explain the $\sim 3.6 \sigma$ discrepancy between the observed and SM value of the muon anomalous magnetic moment $[38,73,74]$ and various dark matter related anomalies via new dark matter- $Z_{D}$ interactions $[75-78]$. Several concrete models have also been suggested in which a sub-GeV mass is generated naturally [33, 79-82], although in many cases masses above $10 \mathrm{GeV}$ are equally natural. However, part of the reason for the attention to sub-GeV masses has been practicality the high-intensity experiments necessary to directly probe dark photons, such as the $B$ and $\Phi$-factories and various fixed-target and beam dump experiments, do not have particle beams with high-enough energy to effectively probe masses above $10 \mathrm{GeV}$.

With the advent of the $14 \mathrm{TeV}$ run at the LHC, including the high-luminosity run (HLLHC), a possible future $100 \mathrm{TeV}$ proton-proton collider, and various possibilities for future electron-positron colliders, we will have the exciting opportunity to probe dark photons well above $10 \mathrm{GeV}$. In fact, these experiments are the only known probe of dark photons above $10 \mathrm{GeV}$ that explore $\epsilon$ values not disfavored by current EWPT. The hypercharge portal allows for direct production of the dark photon in Drell-Yan (DY) events, $p p \rightarrow Z_{D} \rightarrow \ell^{+} \ell^{-}$.

\footnotetext{
${ }^{1}$ Below $1 \mathrm{MeV}$, the dominant decay mode is the long-lived $Z_{D} \rightarrow 3 \gamma$, which leads to a very different phenomenology that we will not discuss in this paper [28-30].
} 

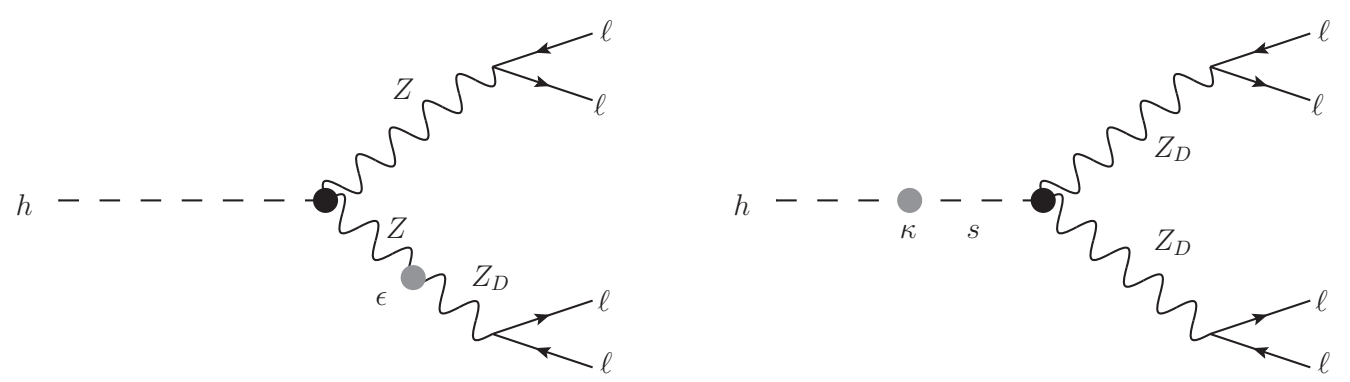

Figure 1. Exotic Higgs decays to four leptons induced by intermediate dark photons in the higgsed dark U(1) model. Left: $h \rightarrow Z_{D} Z^{(*)} \rightarrow 4 \ell$ via the hypercharge portal. Right: $h \rightarrow Z_{D} Z_{D} \rightarrow 4 \ell$ via the Higgs portal.

It also generates the exotic Higgs decay $h \rightarrow Z Z_{D}$. Higgs mixing allows for a different exotic Higgs decay, $h \rightarrow Z_{D} Z_{D}$. Importantly, the Higgs portal can give experimental sensitivity to values of $\epsilon$ far below the reach of searches that only rely on the hypercharge portal, allowing us to peer deeply into the hidden sector.

Existing data from LHC Run I ( 7 and $8 \mathrm{TeV}$ run) are already able to set new limits on dark photons. An initial study in [67] used LHC Run I data to set limits on the exotic Higgs decays $h \rightarrow Z Z_{D} \rightarrow 4 \ell$ and $h \rightarrow Z_{D} Z_{D} \rightarrow 4 \ell$, shown in figure 1 . While the former decay probes a region in the $\epsilon-m_{Z_{D}}$ plane that was already disfavored from EWPTs, the latter generates the first constraints on Higgs portal couplings for dark photon masses above a few $\mathrm{GeV}$. Both analyses are proofs-of-principle that future exotic Higgs decay searches are sensitive to dark photons. Meanwhile, experimental searches for the NMSSM-motivated signal $h \rightarrow a a \rightarrow 4 \mu$, in the region $m_{a}<2 m_{\tau}$, provide limits on Higgs portal couplings for dark photons in the same mass range [83-86] . Other studies [71, 72] pointed out that existing LHC data constrains the production of dark photons in DY events, disfavoring previously open parameter space.

The upcoming HL-LHC and a future $100 \mathrm{TeV}$ collider will significantly extend the sensitivity of these direct searches. Furthermore, the LHC and a future ILC/GigaZ collider will improve the measurement of certain important electroweak precision observables (EWPOs). In this paper, we compare the reach of all these experimental probes. As part of this comparison, we perform a full fit to electroweak precision measurements, presenting a new current bound on dark photons, in addition to forecasting future sensitivity.

The organization of this paper is as follows. Section 2 reviews the theory of a kinetically mixed $\mathrm{U}(1)_{D}$. Sections 3,4 , and 5 analyze existing constraints and future prospects for dark photons being probed via the hypercharge portal only, using EWPOs, the exotic Higgs decay $h \rightarrow Z Z_{D} \rightarrow 4 \ell$, and DY events, respectively. If the dark Higgs mixes with the ordinary Higgs, then the decay $h \rightarrow Z_{D} Z_{D} \rightarrow 4 \ell$ opens up, which we discuss in section 6 . In section 7 we vary the assumed detector capabilities at a future $100 \mathrm{TeV}$ proton collider and discuss the impact this has on our limit projections. Section 8 contains our conclusions. Supplementary information about calculations in the dark photon model are provided by three appendices. 


\section{$2 \quad$ A kinetically mixed dark U(1)}

In this section, we review the theory of kinetic mixing between a broken dark Abelian gauge symmetry, $\mathrm{U}(1)_{D}$, and the SM hypercharge, $\mathrm{U}(1)_{Y}$. The relevant gauge terms in the Lagrangian are

$$
\mathcal{L} \subset-\frac{1}{4} \hat{B}_{\mu \nu} \hat{B}^{\mu \nu}-\frac{1}{4} \hat{Z}_{D \mu \nu} \hat{Z}_{D}^{\mu \nu}+\frac{1}{2} \frac{\epsilon}{\cos \theta} \hat{Z}_{D \mu \nu} \hat{B}^{\mu \nu}+\frac{1}{2} m_{D, 0}^{2} \hat{Z}_{D}^{\mu} \hat{Z}_{D \mu} .
$$

Here the hatted fields indicate the original fields with non-canonical kinetic terms, before any field redefinitions. The $\mathrm{U}(1)_{Y}$ and $\mathrm{U}(1)_{D}$ field strengths are respectively $\hat{B}_{\mu \nu}=\partial_{\mu} \hat{B}_{\nu}-$ $\partial_{\nu} \hat{B}_{\mu}$ and $\hat{Z}_{D \mu \nu}=\partial_{\mu} \hat{Z}_{D \nu}-\partial_{\nu} \hat{Z}_{D \mu}, \theta$ is the Weinberg mixing angle, and $\epsilon$ is the kinetic mixing parameter.

Since the interaction in eq. (2.1) is renormalizable, the parameter $\epsilon$ can take on any value. In particular, $\epsilon$ is not required to be small, which is one reason why the hypercharge portal may provide the dominant interaction between the SM and a hidden sector. Calculable values of $\epsilon$ are obtained in various scenarios. For example, if the $\mathrm{U}(1)_{D}$ is embedded in a Grand Unified Theory (GUT), the mixing is absent above the GUT scale, but can be generated below it by particles charged under both $\mathrm{U}(1)_{Y}$ and $\mathrm{U}(1)_{D}$. If it is generated through a one-(two-)loop interaction, one naturally obtains $\epsilon \sim 10^{-3}-10^{-1}$ $\left(\sim 10^{-5}-10^{-3}\right)[25,79,81,87]$. A much larger range of $\epsilon$ has been suggested in certain string theory scenarios $[28,88-90]$; see [28-30] for recent reviews.

Meanwhile, the general renormalizable potential for the SM and dark Higgs fields is

$$
V_{0}(H, S)=-\mu^{2}|H|^{2}+\lambda|H|^{4}-\mu_{S}^{2}|S|^{2}+\lambda_{S}|S|^{4}+\kappa|S|^{2}|H|^{2} .
$$

Here $H$ is the SM Higgs doublet, while $S$ is the SM-singlet 'dark Higgs' with $\mathrm{U}(1)_{D}$ charge $q_{S}$. The Higgs portal coupling, $\kappa$, which links the dark and SM Higgs fields is again a renormalizable parameter, and may again be sizeable. After spontaneous symmetry breaking in the dark and visible sectors, $\kappa$ controls the mixing between the SM Higgs boson $h_{0}$ and the uneaten component of the dark Higgs, $s_{0}$. The importance of an additional Higgs portal coupling to sectors containing a dark vector boson has been realized before [68, 91], particularly in the context of hidden valley models [92]. While some collider studies have been performed [50,67, 69, 93], its consequences have not been as widely explored as those of the hypercharge portal. The physical dark Higgs boson could in principle be produced at colliders and give an additional experimental handle on the model. However, in this paper we focus on the additional SM Higgs decays to dark photons generated by this interaction, and assume the Higgs decay to dark scalars is kinematically forbidden.

We have also constructed a fully consistent MadGraph 5 [94] implementation of this model using FeynRules 2.0 [95]. This MadGraph model consistently implements all field redefinitions, thereby accurately modeling interference effects, and has been extensively validated by comparing its output to various analytical predictions. We utilize this model in the collider studies of sections 4 and 6 , as well as for the calculation of the three-body decay width $h \rightarrow Z_{D} \ell \ell$ below, and make it publicly available for follow-up investigations. See appendix $C$ for more information. 
The minimal model we consider here can be extended to include strongly-coupled hidden sectors, supersymmetry, and mass mixing, among other possibilities; see e.g. [24, $51,80,96-99]$ for related work. The remainder of this section is devoted to a detailed discussion of the properties of the mass eigenstates in the SM and the hidden sector in the minimal higgsed model, including new results for the branching fractions of the $Z_{D}$.

\subsection{The gauge sector}

We first consider the gauge sector. The field redefinition

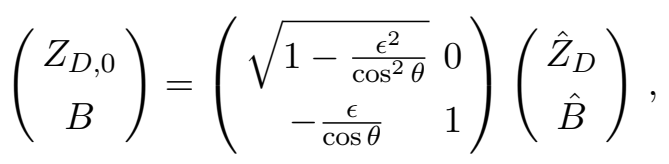

diagonalizes the gauge boson kinetic terms in eq. (2.1) (the subscript ' 0 ' in $Z_{D, 0}$ indicates that this is not yet a mass eigenstate). We define

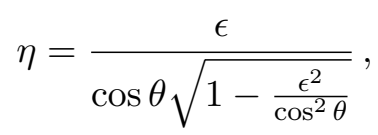

and take the dark vector to have mass $m_{D, 0}^{2} \equiv m_{Z, 0}^{2} \times \delta^{2}$ before mixing with SM fields, where $m_{Z, 0}$ is the mass of the SM $Z$-boson before mixing. After electroweak symmetry breaking (EWSB), and after applying the field redefinition eq. (2.3), we can write the full mass-squared matrix for the three neutral vectors as

$$
\mathcal{M}_{V}^{2}=m_{Z, 0}^{2}\left(\begin{array}{ccc}
0 & 0 & 0 \\
0 & 1 & -\eta \sin \theta \\
0 & -\eta \sin \theta & \eta^{2} \sin ^{2} \theta+\delta^{2}
\end{array}\right)
$$

in the basis $\left(A^{\mu}, Z_{0}^{\mu}, Z_{D, 0}^{\mu}\right)$. Here $A^{\mu}$ is the massless SM photon field and $Z_{0}^{\mu}$ is the SM $Z$-boson field with mass $m_{Z, 0}^{2}=\left(g^{2}+g_{Y}^{2}\right) v^{2} / 4$, where $v \simeq 246 \mathrm{GeV}$ is the SM Higgs vacuum expectation value (vev) and $g\left(g_{Y}\right)$ is the $\mathrm{SU}(2)_{L}\left(\mathrm{U}(1)_{Y}\right)$ gauge coupling. Note that $A^{\mu}$ does not mix with the other neutral fields and remains massless, since electromagnetism remains unbroken. However, the $Z_{0}^{\mu}$ and $Z_{D, 0}^{\mu}$ fields mix, and we can derive the mass eigenstates by diagonalizing the $\left(Z_{0}^{\mu}, Z_{D, 0}^{\mu}\right)$ submatrix of eq. (2.5) with

$$
\left(\begin{array}{c}
Z \\
Z_{D}
\end{array}\right)=\left(\begin{array}{cc}
\cos \alpha & \sin \alpha \\
-\sin \alpha & \cos \alpha
\end{array}\right)\left(\begin{array}{c}
Z_{0} \\
Z_{D, 0}
\end{array}\right),
$$

where the mixing angle is given $b^{2}$

$$
\tan \alpha=\frac{1-\eta^{2} \sin ^{2} \theta-\delta^{2}-\operatorname{Sign}\left(1-\delta^{2}\right) \sqrt{4 \eta^{2} \sin ^{2} \theta+\left(1-\eta^{2} \sin ^{2} \theta-\delta^{2}\right)^{2}}}{2 \eta \sin \theta} .
$$

\footnotetext{
${ }^{2}$ This convention for the mixing angle is chosen so that $\alpha \rightarrow 0$ (not $\pi$ ) when $\epsilon \rightarrow 0$, regardless of whether $m_{Z_{D}}$ is larger or smaller than $m_{Z}$. We make a similar choice when defining the Higgs mixing angle below.
} 
The eigenvalues of the submatrix, in units of $m_{Z, 0}^{2}$, are

$$
m_{Z, Z_{D}}^{2}=\frac{1}{2}\left(1+\delta^{2}+\eta^{2} \sin ^{2} \theta \pm \operatorname{Sign}\left(1-\delta^{2}\right) \sqrt{\left(1+\delta^{2}+\eta^{2} \sin ^{2} \theta\right)^{2}-4 \delta^{2}}\right) .
$$

For $\epsilon \ll 1$ and $\delta \ll 1$, the masses are $m_{Z_{D}}^{2} \simeq \delta^{2} m_{Z, 0}^{2}\left(1-\epsilon^{2} \tan ^{2} \theta\right)$ and $m_{Z}^{2} \simeq$ $m_{Z, 0}^{2}\left(1+\epsilon^{2} \tan ^{2} \theta\right)$.

Having written the theory in terms of canonically normalized kinetic terms and mass eigenstates, several important consequences become apparent. The interaction between the $Z$-boson and the SM fermions, $Z \bar{f} f$, has been modified from the SM expectation,

$$
\begin{aligned}
\mathcal{L}_{Z \bar{f} f} & =g_{Z f \bar{f}} Z_{\mu} \bar{f} \gamma^{\mu} f \\
g_{Z f \bar{f}} & \equiv \frac{g}{\cos \theta}\left(\cos \alpha\left(t^{3} \cos ^{2} \theta-Y \sin ^{2} \theta\right)+\eta \sin \alpha \sin \theta Y\right),
\end{aligned}
$$

where $t^{3}$ and $Y$ are the weak isospin and hypercharge value, respectively, of the fermion $f$. The $Z_{D} \bar{f} f$ interaction is non-zero,

$$
\begin{aligned}
\mathcal{L}_{Z_{D} \bar{f} f} & =g_{Z_{D} f \bar{f}} Z_{D, \mu} \bar{f} \gamma^{\mu} f \\
g_{Z_{D} f \bar{f}} & \equiv \frac{g}{\cos \theta}\left(-\sin \alpha\left(t^{3} \cos ^{2} \theta-Y \sin ^{2} \theta\right)+\eta \cos \alpha \sin \theta Y\right) .
\end{aligned}
$$

For $\epsilon \ll 1$, at leading order, the $Z_{D}$ coupling to fermions is "photon-like" for $\delta \ll 1$ : $g_{Z_{D} f \bar{f}} \simeq \epsilon e Q+\mathcal{O}\left(\delta^{2}\right)$, where $e=\sqrt{4 \pi \alpha}$ is the electromagnetic coupling and $Q$ the fermion charge, and "Z-like" for $|\delta| \simeq 1: g_{Z_{D} f \bar{f}} \simeq \epsilon \frac{g}{\cos \theta}\left(t^{3} \cos ^{2} \theta-Y \sin ^{2} \theta\right)$. Furthermore, the interaction $Z \bar{f} f$ receives its first correction at $\mathcal{O}\left(\epsilon^{2}\right)$, given by $g_{Z \bar{f} f} \simeq g_{Z \bar{f} f}^{\mathrm{SM}}+\epsilon^{2} \frac{\tan ^{2} \theta}{2} \frac{g}{\cos \theta}\left(t^{3}-\right.$ $\left.Q\left(1+\cos ^{2} \theta\right)+2 Y \delta^{2}\right) /\left(1-\delta^{2}\right)^{2}$. The admixture of the $Z$-boson in the $Z_{D}$ mass eigenstate gives rise to a coupling between the SM Higgs boson to $Z$ and $Z_{D}$ after EWSB,

$$
\begin{aligned}
\mathcal{L}_{h Z Z_{D}} & =\left[\frac{2 i \eta \sin \theta}{v} m_{Z_{0}}^{2}\left(\frac{\eta^{2} \sin ^{2} \theta-1}{\eta \sin \theta} 2 \sin 2 \alpha-\cos 2 \alpha\right)\right] h Z_{\mu} Z_{D}^{\mu} \\
& =\frac{2 i \eta \sin \theta}{v} \frac{m_{Z_{D}}^{2} m_{Z}^{2}}{m_{Z}^{2}-m_{Z_{D}}^{2}} h Z_{\mu} Z_{D}^{\mu}+\mathcal{O}\left(\eta^{3}\right),
\end{aligned}
$$

where, again, $m_{Z, 0}$ is the mass of the $Z$ before mixing, and $m_{Z, Z_{D}}$ are the physical $Z, Z_{D}$ masses. At $\mathcal{O}\left(\epsilon^{2}\right)$, this vertex mediates both (i) the decay of the Higgs to a (potentially off-shell) $Z$ and an on-shell $Z_{D}$, and (ii) interference from an off-shell $Z_{D}$ in the decay $h \rightarrow Z^{(*)} Z^{*} \rightarrow 4 f$. Sensitivity to $Z_{D}$ will come almost entirely from its production on-shell, and thus we ignore the interference contributions in our collider studies below. However, post-discovery, the $Z_{D}$ interference terms in Higgs decays to four leptons may present a unique opportunity to distinguish the sign of $\epsilon$, though this would, of course, require much larger integrated luminosities than those needed for discovery.

Note that the overall rate for the SM decay $h \rightarrow Z Z^{*} \rightarrow 4 f$ is also modified at $\mathcal{O}\left(\epsilon^{2}\right)$, owing to the modifications of the $Z$ mass, $h Z Z$ vertex, and $Z \bar{f} f$ couplings, all of which receive $\mathcal{O}\left(\epsilon^{2}\right)$ contributions. However, this effect is simply an overall numerical suppression of the rate, and does not change the shape of any lepton distributions. Due to its small 
size, it is therefore not observable in the currently forseeable future, given the theoretical uncertainties on the SM branching fractions $\operatorname{Br}(h \rightarrow c \bar{c})$ and $\operatorname{Br}(h \rightarrow b \bar{b})$, with additional limitations from experimental precision in the determination of $\operatorname{Br}\left(h \rightarrow Z Z^{*}\right)$. For this reason we do not consider these contributions further.

We first discuss dark photon decays, since this affects the experimental signatures of all exotic Higgs decay modes under consideration in this paper. The lowest order (LO) dark photon decay widths are

$$
\Gamma\left(Z_{D} \rightarrow \bar{f} f\right)=\frac{N_{c}}{24 \pi m_{Z_{D}}} \sqrt{1-\frac{4 m_{f}^{2}}{m_{Z_{D}}^{2}}}\left(m_{Z_{D}}^{2}\left(g_{L}^{2}+g_{R}^{2}\right)-m_{f}^{2}\left(-6 g_{L} g_{R}+g_{L}^{2}+g_{R}^{2}\right)\right)
$$

where $g_{L, R}=g_{Z_{D} f_{L, R} \bar{f}_{L, R}}$ are given in eq. (2.10) and are proportional to $\epsilon$ for $\epsilon \ll 1$. This tree-level parton-level formula is a good approximation for $m_{Z_{D}}$ above the $b \bar{b}$ threshold. For smaller masses, threshold effects, QCD corrections, and hadronic resonances cannot be neglected. To obtain consistent predictions for the dark photon total width and branching fractions across the entire relevant mass range we must include experimental information and higher order QCD calculations.

Define the ratio

$$
R_{Z_{D}} \equiv \frac{\Gamma\left(Z_{D} \rightarrow \text { hadrons }\right)}{\Gamma\left(Z_{D} \rightarrow \mu^{+} \mu^{-}\right)} \underset{\epsilon \ll 1}{=} R_{Z_{D}}\left(m_{Z_{D}}\right)
$$

which is independent of $\epsilon$ for $\epsilon \ll 1$. If we knew this function, including higher order corrections, we could write the total width of $Z_{D}$ to high accuracy as

$$
\Gamma_{Z_{D}}=R_{Z_{D}} \Gamma\left(Z_{D} \rightarrow \mu^{+} \mu^{-}\right)+\sum_{f=e, \mu, \tau, \nu_{e}, \mu, \tau} \Gamma\left(Z_{D} \rightarrow f \bar{f}\right)
$$

where all the partial widths are computed at LO using eq. (2.12). This also gives the leptonic branching fractions

$$
\operatorname{Br}\left(Z_{D} \rightarrow \ell \ell\right)=\frac{\Gamma\left(Z_{D} \rightarrow \ell \ell\right)}{\Gamma_{Z_{D}}}
$$

to high accuracy.

In fact, we can obtain $R_{Z_{D}}\left(m_{Z_{D}}\right)$ very accurately. For $m_{Z_{D}}<12 \mathrm{GeV}$, the couplings of $Z_{D}$ to SM fermions are photon-like up to corrections of order $\delta^{2}(<2 \%)$. Furthermore, for $\sqrt{s} \ll m_{Z}^{2}$, the experimental ratio

$$
R(s) \equiv \frac{\sigma\left(e^{+} e^{-} \rightarrow \text { hadrons }\right)}{\sigma\left(e^{+} e^{-} \rightarrow \mu^{+} \mu^{-}\right)}
$$

is highly dominated by off-shell $\gamma^{*} \rightarrow f \bar{f}$ in the $s$-channel. Therefore we can use experimental data [100] to determine

$$
R_{Z_{D}}\left(m_{Z_{D}}\right)=R\left(m_{Z_{D}}^{2}\right) \quad \text { for } \quad m_{Z_{D}}<12 \mathrm{GeV}
$$



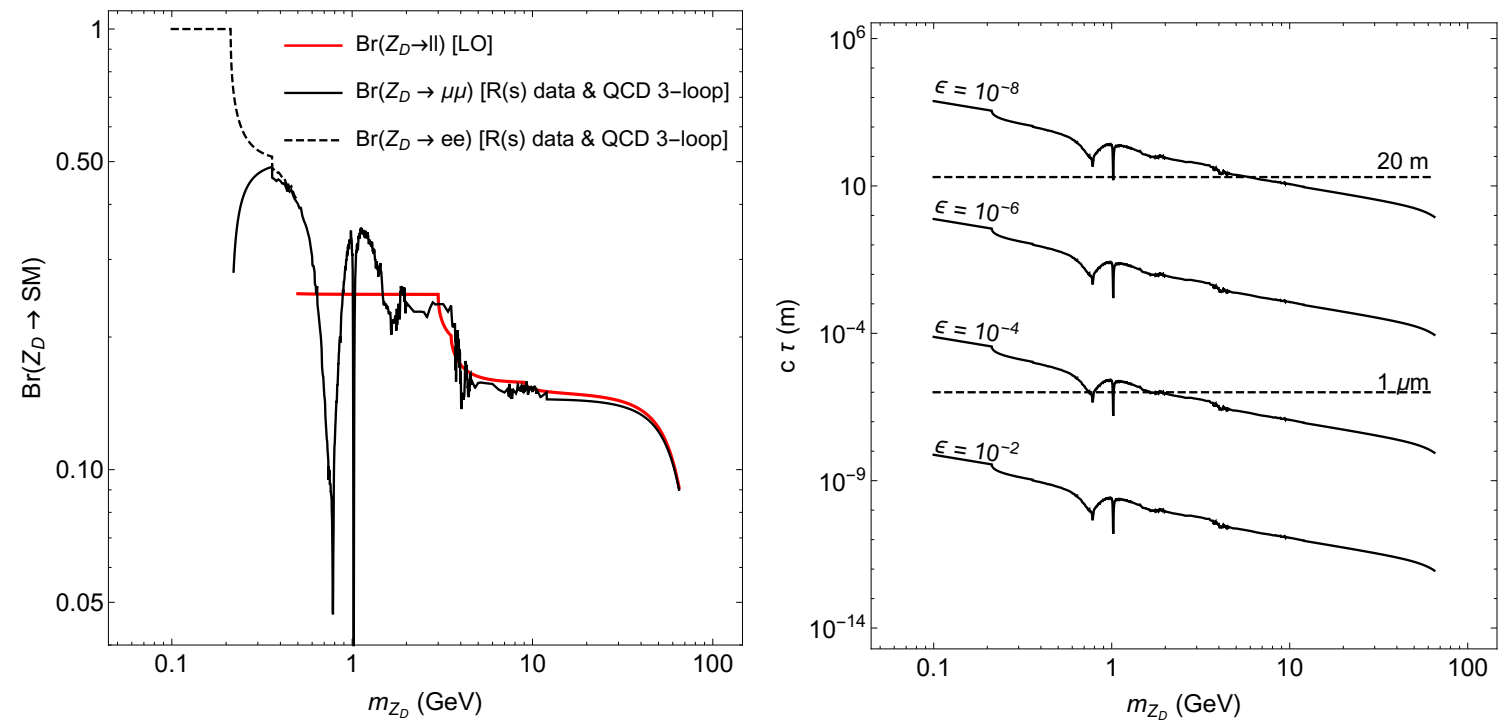

Figure 2. Left: leptonic branching fraction of $Z_{D}$. Right: decay length of $Z_{D}$ for different $\epsilon$. The dashed lines indicate boundaries between qualitatively different experimental regimes: prompt decay for $c \tau \lesssim 1 \mu \mathrm{m}$ and likely escape from an ATLAS-size detector for $c \tau \gtrsim 20 \mathrm{~m}$.

which includes all higher order QCD corrections. ${ }^{3}$ For higher masses, the $Z_{D}$ couplings are different from that of the photon. In this regime, we use existing 3-loop QCD calculations of $R(s)$ [101] to compute $R_{Z_{D}}$ by replacing the SM coupling between the (axial) vector current and quarks by the $Z_{D}$ couplings in eq. (2.10). ${ }^{4}$ In the notation of [101], we can then determine

$$
R_{Z_{D}}\left(m_{Z_{D}}\right)=\frac{\left[R^{(v)}+R^{(a)}\right]_{\text {hadrons }}}{\left[R^{(v)}+R^{(a)}\right]_{\mu \mu}} \quad \text { for } \quad m_{Z_{D}}>12 \mathrm{GeV},
$$

where the running QCD coupling was computed at 3+ loop order using the RunDec Mathematica package [103]. The resulting leptonic branching fraction and total width of the dark photon are shown in figure 2. We will use these high-precision results throughout the paper, but, as the figure shows, the LO expression for total width and leptonic branching fraction is an excellent approximation at higher masses: the higher order corrections are $4 \%$ $(1.5 \%)$ at $m_{Z_{D}}=12 \mathrm{GeV}(60 \mathrm{GeV})$. See appendix A for tables of these branching ratios.

The above interactions eqs. (2.10) and (2.11) allow the decay $h \rightarrow Z_{D} Z^{(*)} \rightarrow 4 \ell$, shown in figure 1 (left). The partial width for the exotic two-body decay $h \rightarrow Z Z_{D}$ is

$$
\begin{aligned}
\Gamma\left(h \rightarrow Z Z_{D}\right)= & \frac{\eta^{2} \sin ^{2} \theta m_{Z}^{2} m_{Z_{D}}^{2}}{16 \pi v^{2} m_{h}^{3}\left(m_{Z}^{2}-m_{Z_{D}}^{2}\right)^{2}}\left(-2 m_{Z_{D}}^{2}\left(m_{h}^{2}-5 m_{Z}^{2}\right)+m_{Z_{D}}^{4}+\left(m_{h}^{2}-m_{Z}^{2}\right)^{2}\right) \\
& \times \sqrt{-2 m_{h}^{2}\left(m_{Z_{D}}^{2}+m_{Z}^{2}\right)+\left(m_{Z}^{2}-m_{Z_{D}}^{2}\right)^{2}+m_{h}^{4}} .
\end{aligned}
$$

\footnotetext{
${ }^{3}$ There is no data below $\sqrt{s}=0.36 \mathrm{GeV}$, so for $2 m_{\pi}<m_{Z_{D}}<0.36 \mathrm{GeV}$ we set $R=0$. This does not affect the results we derive in this paper.

${ }^{4}$ See $[102]$ for a general review on these computations.
} 
The partial width for the three-body decay $h \rightarrow Z_{D} Z^{*} \rightarrow Z_{D} \ell \ell$ is, to leading order in $\epsilon$,

$$
\Gamma\left(h \rightarrow Z_{D} Z^{(*)} \rightarrow 4 \ell\right)=\frac{\eta^{2} \sin ^{2} \theta}{64 \pi^{3}} \frac{m_{Z}^{4}}{m_{h}^{3} v^{2}}\left(g_{Z, L}^{2}+g_{Z, R}^{2}\right)\left(\frac{\delta^{2}}{1-\delta^{2}}\right)^{2} \mathcal{I}\left(m_{Z}, m_{h}, m_{Z_{D}}\right),
$$

where

$$
\begin{gathered}
\mathcal{I}\left(m_{Z}, m_{h}, m_{Z_{D}}\right) \equiv \int_{0}^{\left(m_{h}-m_{Z_{D}}\right)^{2}} d w \frac{\left(m_{Z_{D}}^{4}-2 m_{Z_{D}}^{2}\left(m_{h}^{2}-5 w\right)+\left(m_{h}^{2}-w\right)^{2}\right)}{6 m_{Z_{D}}^{2}\left(m_{Z}^{2}-w\right)^{2}} \\
\times \sqrt{m_{Z_{D}}^{4}+\left(m_{h}^{2}-w\right)^{2}-2 m_{Z_{D}}^{2}\left(m_{h}^{2}+w\right)},
\end{gathered}
$$

and $g_{Z, L}, g_{Z, R}$ are the (tree-level) couplings of a lepton to the $Z$ boson, as in eq. (2.9). For $m_{Z_{D}} \sim m_{h}-m_{Z}$, finite-width effects of the $Z$ are most easily accounted for by computing the partial width in MadGraph. figure 3 (top) shows $\operatorname{Br}\left(h \rightarrow Z_{D} Z^{(*)} \rightarrow 4 \ell\right.$ ) for different values of $\epsilon$.

We note that the kinetic mixing interaction by itself also generates the decay $h \rightarrow$ $Z_{D} Z_{D}$. This decay is highly suppressed, as it requires that both $Z$ 's in $h \rightarrow Z Z^{(*)}$ mix with the $Z_{D}$, see e.g. [67], and appears first at $\mathcal{O}\left(\epsilon^{4}\right)$. However, if the SM Higgs mixes with the hidden-sector Higgs, then this decay can proceed through Higgs portal mixing instead, allowing it to be potentially sizable, as we will now discuss below.

\subsection{The Higgs sector}

We now consider the Higgs sector. Electroweak symmetry is broken by $\langle H\rangle=(0, v / \sqrt{2})$, where $v \approx 246 \mathrm{GeV}$. The singlet acquires a vev $\langle S\rangle=v_{S} / \sqrt{2}$, which generates the dark photon mass of eq. (2.1):

$$
m_{D, 0}=g_{D} q_{S} v_{S} .
$$

Rewriting the scalar mass terms eq. (2.2) in terms of these vevs gives

$$
\mu^{2}=v^{2} \lambda+\frac{1}{2} \kappa v_{S}^{2}, \quad \mu_{S}^{2}=v_{S}^{2} \lambda_{S}+\frac{1}{2} \kappa v^{2} .
$$

Expanding in small fluctuations $h_{0}, s_{0}$ (not yet mass eigenstates) around the vacuum, the Higgs mass matrix in the $\left(h_{0}, s_{0}\right)$ basis is

$$
M_{h_{0} s_{0}}^{2}=\left(\begin{array}{cc}
2 v^{2} \lambda & v v_{S} \kappa \\
v v_{S} \kappa & 2 v_{S}^{2} \lambda_{S}
\end{array}\right)
$$

We define mass eigenstates $(h, s)$

$$
\left(\begin{array}{l}
h \\
s
\end{array}\right)=\left(\begin{array}{cc}
\cos \theta_{h} & -\sin \theta_{h} \\
\sin \theta_{h} & \cos \theta_{h}
\end{array}\right)\left(\begin{array}{l}
h_{0} \\
s_{0}
\end{array}\right)
$$

(note the minus sign). For small mixing angles, $h$ is dominantly SM-Higgs-like and $s$ is dominantly singlet-Higgs like. The mixing angle is given by

$$
\tan \theta_{h}=\frac{v^{2} \lambda-v_{S}^{2} \lambda_{S}-\operatorname{Sign}\left(v^{2} \lambda-v_{S}^{2} \lambda_{S}\right) \sqrt{v^{4} \lambda^{2}+v_{S}^{4} \lambda_{S}^{2}+v^{2} v_{S}^{2}\left(\kappa^{2}-2 \lambda \lambda_{S}\right)}}{v v_{S} \kappa} .
$$



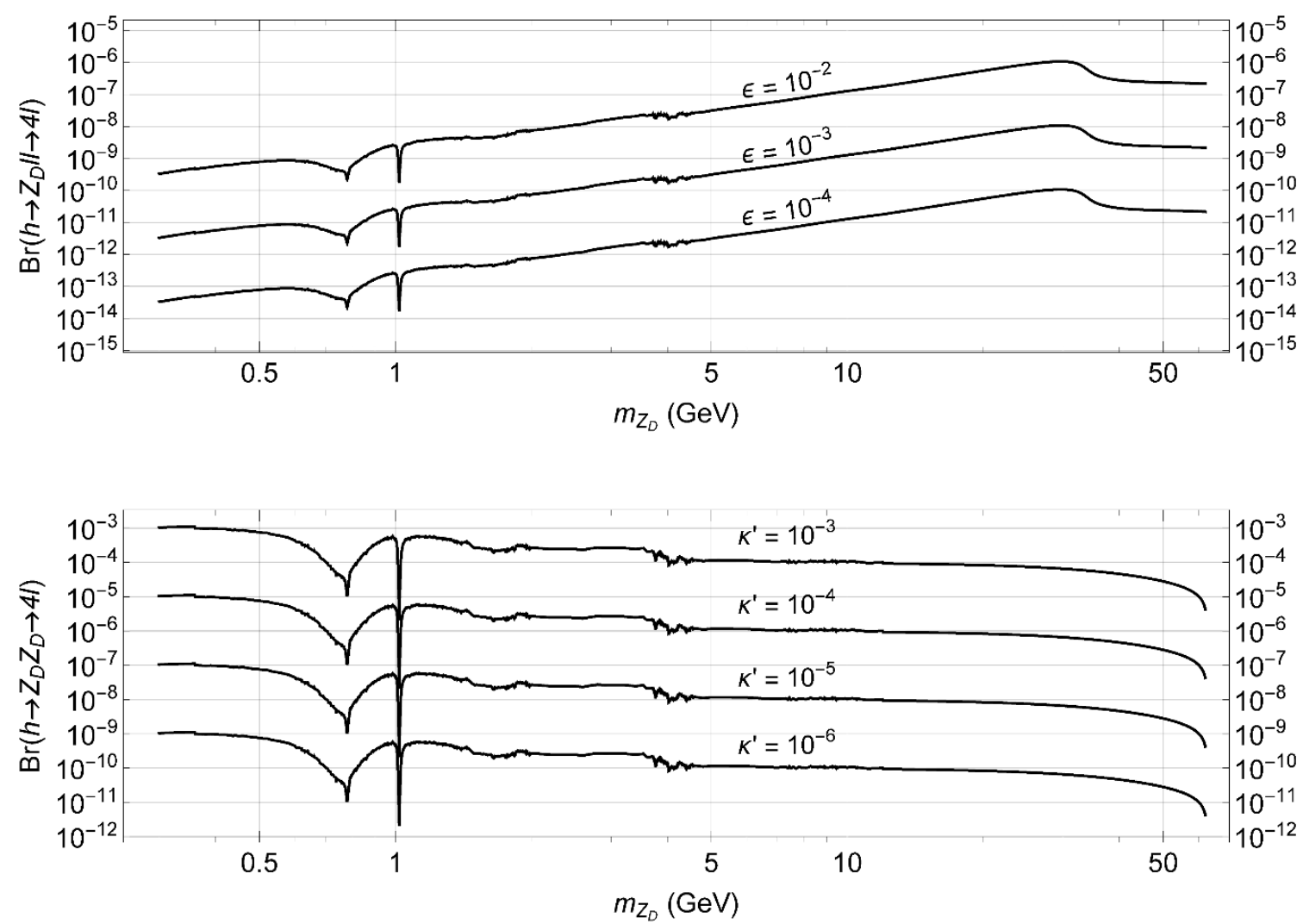

Figure 3. $\operatorname{Br}\left(h \rightarrow Z_{D} Z^{*} \rightarrow 4 \ell\right)($ top $)$ and $\operatorname{Br}\left(h \rightarrow Z_{D} Z_{D} \rightarrow 4 \ell\right.$ ) (bottom) for different values of $\epsilon$ and $\kappa^{\prime}$.

If we define

$$
s_{h} \equiv \frac{\kappa}{2} \frac{v v_{S}}{v_{S}^{2} \lambda_{S}-v^{2} \lambda}
$$

then for small Higgs mixing,

$$
\tan \theta_{h} \approx \sin \theta_{h}=s_{h}+\mathcal{O}\left(\kappa^{2}\right) .
$$

The mass eigenvalues are

$$
m_{h, s}^{2}=v^{2} \lambda+v_{S}^{2} \lambda_{S} \pm \operatorname{Sign}\left(v^{2} \lambda-v_{S}^{2} \lambda_{S}\right) \sqrt{v^{4} \lambda^{2}+v_{S}^{4} \lambda_{S}^{2}+v^{2} v_{S}^{2}\left(\kappa^{2}-2 \lambda \lambda_{S}\right)} .
$$

For small Higgs mixing, this reduces to

$$
\begin{aligned}
& m_{h}^{2}=2 \lambda v^{2}+2 s_{h}^{2}\left(\lambda v^{2}-\lambda_{S} v_{S}^{2}\right)+\mathcal{O}\left(\kappa^{4}\right) \\
& m_{s}^{2}=2 \lambda_{S} v_{S}^{2}-2 s_{h}^{2}\left(\lambda v^{2}-\lambda_{S} v_{S}^{2}\right)+\mathcal{O}\left(\kappa^{4}\right) .
\end{aligned}
$$

Since the $s Z_{D} Z_{D}$ coupling is non-zero $\left(=2 g_{D} q_{S} m_{Z_{D}}\right)$, the mixing between $h$ and $s$ generates a non-zero $h Z_{D} Z_{D}$ coupling. To lowest order in $\kappa$, this is

$$
\mathcal{L}_{h Z_{D} Z_{D}}=2 s_{h} \frac{m_{Z_{D}}^{2}}{v_{s}} h Z_{D \mu} Z_{D}^{\mu}
$$


This allows for the decay $h \rightarrow Z_{D} Z_{D}$, shown in figure 1 (right). The partial width to lowest order in $\kappa$ is

$$
\Gamma\left(h \rightarrow Z_{D} Z_{D}\right)=\kappa^{\prime 2} \frac{1}{32 \pi} \frac{v^{2}}{m_{h}} \sqrt{1-\frac{4 m_{Z_{D}}^{2}}{m_{h}^{2}}} \frac{\left(m_{h}^{2}+2 m_{Z_{D}}^{2}\right)^{2}-8\left(m_{h}^{2}-m_{Z_{D}}^{2}\right) m_{Z_{D}}^{2}}{m_{h}^{4}},
$$

where we have have introduced the dimensionless parameter $\kappa^{\prime}$, defined as

$$
\kappa^{\prime}=\kappa \frac{m_{h}^{2}}{\left|m_{h}^{2}-m_{s}^{2}\right|},
$$

which, along with $m_{Z_{D}}$, controls the size of this exotic Higgs decay. The resulting $\operatorname{Br}(h \rightarrow$ $Z_{D} Z_{D} \rightarrow 4 \ell$ ) is shown in figure 3 (bottom) for different values of $\kappa^{\prime}$. It does not depend on $\epsilon$, but the decay length of the dark photons does.

An additional interaction exists that allows for $h \rightarrow s s$. We will simply assume that $s$ is heavy enough that this decay is kinematically forbidden, but see e.g. [67] for a more comprehensive discussion of the several possibilities. One can also produce the singlet scalar directly via its inherited SM couplings, analogously to the SM Higgs boson. The dominant mode for $m_{s}>m_{h} / 2$ is gluon fusion, but as we discuss in Section 6, the Higgs portal is more sensitively probed by $p p \rightarrow h \rightarrow Z_{D} Z_{D}$ than by $p p \rightarrow s \rightarrow Z_{D} Z_{D}$, even though both processes occur at the same order in $\kappa$. The singlet scalar can also be produced via the process $p p \rightarrow Z_{D}^{*} \rightarrow Z_{D} s$, which occurs at the same order of $\epsilon$ as the exotic Higgs decay $h \rightarrow Z Z_{D}$. All of these channels should be studied more comprehensively in the future, but are beyond the scope of this paper.

As demonstrated in figure 3 , the $\operatorname{Br}\left(h \rightarrow Z_{D} Z_{D}\right)$ can be quite sizable. However, this decay is invisible unless $Z_{D}$ decays inside the detector, and therefore $\epsilon$ cannot be too small in order for this Higgs portal decay to be observable. A large fraction of $Z_{D}$ will decay inside the detector for $\epsilon \gtrsim 10^{-7}$ (see right panel of figure 2), but the large luminosity of hadron colliders means that even $\epsilon \sim 10^{-10}$ could be detected by looking for two displaced $Z_{D} \rightarrow \ell \ell$ decays. This presents us with the exciting opportunity to probe very small values of $\epsilon$ if some Higgs mixing is present, as we discuss in section 6 .

\section{Constraining the hypercharge portal with electroweak precision ob- servables}

The discovery of a light Higgs boson has been an excellent confirmation of the selfconsistency of the electroweak sector of the SM. In fact, global fits of electroweak precision observables measured at lepton (LEP, SLC) and hadron (Tevatron, LHC) colliders show that the SM provides a good fit to the data, with a $p$-value of $\sim 0.2$ [104] (see also [105108] for earlier fits post-Higgs-discovery). Measurements of the various EWPOs are in good agreement with the SM prediction, with the exception of the notorious forward-backward asymmetry of the bottom quark, $A_{F B}^{b, 0}$, as measured at the Z-pole at LEP1, which differs by $\sim 2.5 \sigma$ from the SM prediction.

In this context, physics beyond the SM can receive important constraints from EWPOs. In particular, here we investigate the bound on the hypercharge portal coupling, given by 
eq. (2.1), that can be obtained from electroweak precision measurements. (Constraints from EWPOs on the Higgs portal eq. (2.2) are unimportant, and we thus do not consider them.) We will perform a fit to the current measurements of EWPOs and also consider the impact of future improvements from hadron and lepton colliders.

In contrast to [41], our approach closely mirrors the procedure performed by the Gfitter group [109], and introduces all observables directly related to properties of the electroweak bosons, including observables that are not corrected at tree level in the dark photon model, such as $m_{W}$. As we will see, the precision that will be available in future experimental determinations of $m_{W}$ will make $m_{W}$ one of the main drivers in future electroweak fits. To begin, we implement the SM fit to the EWPO data. We consider the following set of independent observables:

$$
\begin{gathered}
m_{Z}, \Gamma_{Z}, \sigma_{\text {had }}^{0}, R_{\ell}^{0}, R_{c}^{0}, R_{b}^{0}, A_{F B}^{\ell, 0}, A_{\ell}, A_{c}, A_{b}, A_{F B}^{c, 0}, A_{F B}^{b, 0}, \sin ^{2} \theta_{\text {eff }}^{\ell}\left(Q_{\mathrm{FB}}\right), \\
m_{W}, \Gamma_{W}, m_{t}, \Delta \alpha_{\text {had }}^{(5)}, m_{h},
\end{gathered}
$$

The experimental measurements of these observables are tabulated in [104] (see also appendix B for a summary), whose approach to the data we largely follow. Note that this fit makes use of the inclusive hadronic charge asymmetry measurements of $\sin ^{2} \theta_{\text {eff }}$, which we call $\sin ^{2} \theta_{\mathrm{eff}}^{\ell}\left(Q_{\mathrm{FB}}\right)$. We include this observable to verify our procedure against the GFitter results. However, we will not use $\sin ^{2} \theta_{\text {eff }}^{\ell}\left(Q_{\mathrm{FB}}\right)$ to obtain bounds on the dark photon model, since this measurement is difficult to interpret in theories with vertex corrections to the $Z$ boson coupling (see also [110]).

A convenient set of independent input observables is

$$
m_{h}, m_{Z}, m_{t}, \alpha_{s}, \Delta \alpha_{\text {had }}^{(5)},
$$

the latter of which replaces the electromagnetic coupling $\alpha\left(m_{Z}^{2}\right)$ and is related to the strong coupling constant $\alpha_{s}\left(m_{Z}\right)$. The light quark masses and the Fermi constant, $G_{F}$, should in principle also be added to the set of input observables. Since $G_{F}$ is very precisely determined from muon decay measurements, we simply fix it to its measured value. Likewise, the pole masses for $m_{b}$ and $m_{c}$ are very well determined, and the difference between the pole mass and the less well-determined running mass enters at higher order and makes a negligible contribution to the fit. Therefore, we simply fix $m_{b}, m_{c}$ to their $\overline{\mathrm{MS}}$ masses. We set the light quark masses $m_{u}, m_{d}$, and $m_{s}$ to their world averages. We refer to [111-114] for the SM prediction of the $W$ boson mass, $Z$ boson partial widths ${ }^{5}$ (see also appendix B for more details), the effective mixing angle $\sin ^{2} \theta_{\mathrm{eff}}^{\ell}$, and $R_{b}^{0}$, respectively. We build our log-likelihood function through the comparison between the SM prediction and the corresponding measurement, taking into account the correlation matrices among the $Z$-lineshape and the heavy-flavor observables,

$$
\chi_{\mathrm{SM}}^{2}=V_{\mathrm{SM}} \cdot \operatorname{cov}^{-1} \cdot V_{\mathrm{SM}} \text { with } \operatorname{cov}=\Sigma_{\exp } \cdot \operatorname{cor} \cdot \Sigma_{\mathrm{exp}},
$$

\footnotetext{
${ }^{5}$ We do not include the full fermionic two-loop corrections $\mathcal{O}\left(\alpha^{2}\right)$ to the $Z$ partial widths, as recently computed in [115]. Given the numerically small effects of these corrections on the SM fit [104], we do not anticipate these corrections to substantially impact our results.
} 
where $V_{\mathrm{SM}}$ is the difference vector between the SM prediction and the experimentally measured value of the observables in (3.1): $V_{\mathrm{SM}}=$ theory $_{\mathrm{SM}}\left(m_{h}, m_{Z}, m_{t}, \alpha_{s}, \Delta \alpha_{\text {had }}^{(5)}\right)-\exp$. Meanwhile, $\Sigma_{\exp }$ is the vector containing the experimental error on the corresponding measurements, and the correlation matrix cor can be found in [116]. In this definition of $\chi^{2}$, we neglect the theoretical uncertainties in the determination of the various observables. This is a good approximation for all observables except the top mass, for which the experimental uncertainty $\left(\delta m_{t}^{\exp }=0.76 \mathrm{GeV}\right)$ is comparable to the theory uncertainty (see [117] for a detailed discussion of the latter). The value of the input observables in (3.2) is varied around the measured value to minimize the $\chi^{2}$. The resulting $p$-value that we obtain for the $\mathrm{SM}$ is very similar to the one obtained by [104]: $\chi_{S M}^{2} /$ d.o.f. $=16.7 / 13$ corresponding to a p-value $p_{S M}=0.21$. Dropping the $\sin ^{2} \theta_{\text {eff }}^{\ell}\left(Q_{\mathrm{FB}}\right)$ measurement gives a very similar result, though the fit is slightly worse, with $\chi_{S M}^{2 \prime} /$ d.o.f. $=16.1 / 12$, corresponding to a p-value $p_{S M}^{\prime}=0.19$.

We now consider the effect of adding a dark photon. This introduces two independent effects in the electroweak fit:

- a shift in the $Z$ mass observable [41, 68], see eq. (2.8), from its input value in eq. (3.2). The input value is what we call $m_{Z, 0}$ in eq. (2.5). This effect also induces a small correction to the $Z$ total width and to the hadronic peak cross section, $\sigma_{\text {had }}^{0}$ purely through kinematics;

- a shift of the $Z$ couplings to SM fermions, see eq. (2.9), and, consequently, a new physics effect on the heavy-flavor observables, as well as on $\Gamma_{Z}, \sigma_{\text {had }}^{0}, R_{\ell}^{0}, A_{\ell}$, and $A_{F B}^{\ell, 0}$.

Both effects first appear at $\mathcal{O}\left(\epsilon^{2}\right)$ and are therefore independent of the sign of $\epsilon$. Further details are included in appendix B. Note again that $\sin ^{2} \theta_{\mathrm{eff}}^{\ell}\left(Q_{\mathrm{FB}}\right)$ is not used in this fit.

Analogously to the fit we perform for the SM, we build the $\chi^{2}$ for the theory of a kinetically mixed $\mathrm{U}(1)$, denoted $\chi_{Z_{D}}^{2}$, and compare the results to the goodness of the fit obtained for the SM. The coupling $\epsilon$ can be constrained by imposing an upper bound on $\chi_{Z_{D}}^{2}$,

$$
\chi_{Z_{D}}^{2}-\chi_{S M}^{2 \prime} \lesssim 3.8
$$

corresponding to a $95 \%$ CL bound in the case of one degree of freedom (the $\epsilon$ parameter, once the $Z_{D}$ mass has been fixed). Note that we have chosen to present a bound requiring that the deviation of the dark photon model from the SM not exceed $\approx 2 \sigma$, rather than imposing $\chi_{Z_{D}}^{2} \lesssim 3.8$ on the dark photon model alone. This is in order to avoid overinterpreting the $\sim 1 \sigma$ tension between the SM predictions and the experimental measurements, which is largely driven by $A_{F B}^{b, 0}$. While $A_{F B}^{b, 0}$ is shifted in the dark photon model, other observables, such as leptonic asymmetries, receive comparable shifts, and those observables show no significant deviation between experimental measurement and SM predictions. Kinetic mixing therefore does not preferentially ameliorate the most significant pull in the SM fit.

The solid purple line in figure 4 shows the bound obtained by imposing the requirement of eq. (3.4). This is interpreted as the current upper limit on the size of $\epsilon$. For comparison, the green solid line in figure 4 shows the $95 \%$ CL limit on $\epsilon$ that is obtained from eq. (3.4) if we artificially adjust the central values of the EWPOs to the values that give the best 


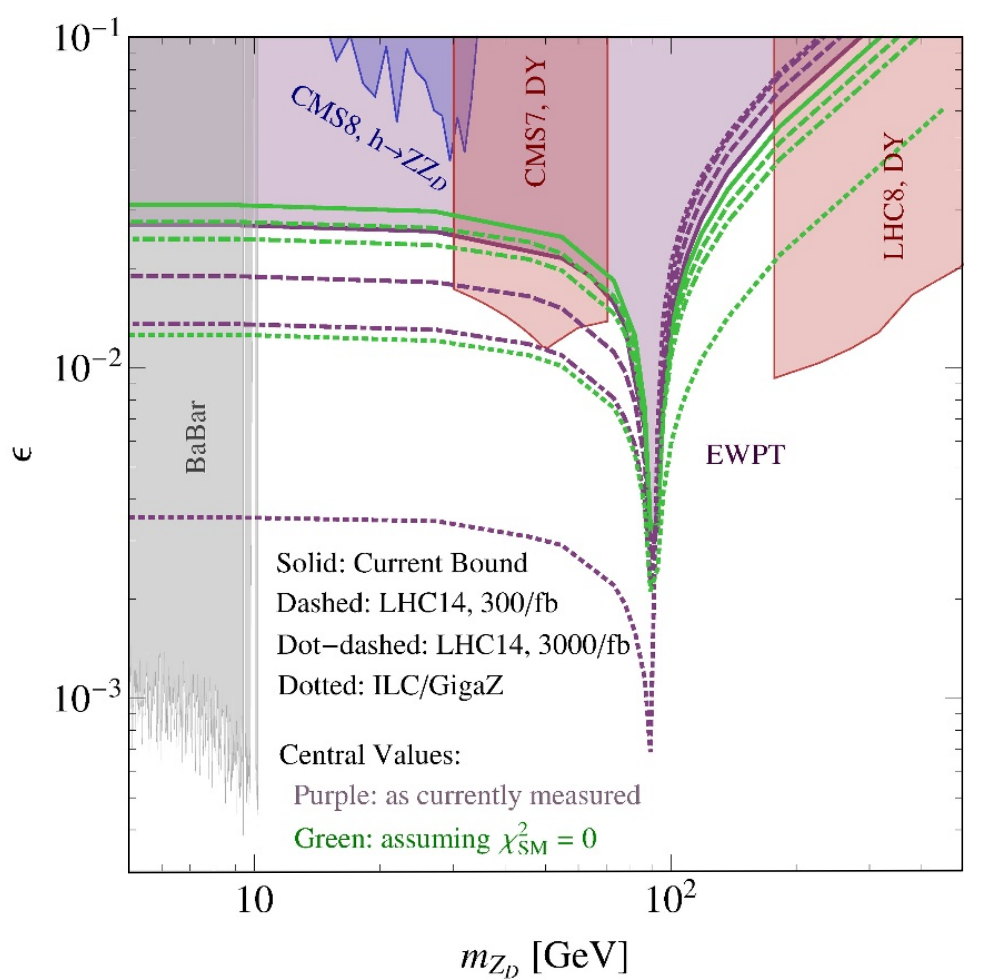

Figure 4. Present bound (purple shaded region) on the kinetic mixing coefficient $\epsilon$ from the fit to electroweak precision observables. Future projected reach at the $14 \mathrm{TeV} \mathrm{LHC}$ with $300 \mathrm{fb}^{-1} \mathrm{and}$ $3000 \mathrm{fb}^{-1}$ of data, and at the ILC/GigaZ are shown by the dashed, dot-dashed, and dotted lines, respectively. Purple and green lines, respectively, represent the bounds obtained by keeping the central values of the measurements as they are now, or with central values adjusted to the values predicted by the SM best fit. For the ILC/GigaZ bound, we also assume the $14 \mathrm{TeV}$ LHC (3000 $\mathrm{fb}^{-1}$ data) precision measurements of $m_{h}$ and $m_{t}$. The HL-LHC and ILC/GigaZ projections also include expected improvements in the measurement of $\Delta \alpha_{\text {had }}^{(5)}$ from VEPP-2000/Babar data.

fit to the SM. Above the $Z$ mass the latter fit (in green) gives slightly stronger bounds on $\epsilon$ than the fit obtained keeping the central values of the measurements as they are now (in purple). This is because, for sufficiently small values of $\epsilon$, the effect of the dark photon improves the electroweak fit. The opposite is true below the $Z$ mass. The most important pulls for our theory are the $W$ boson mass, which is the next-best measured observable after the $Z$ boson mass, and the asymmetry parameter $A_{\ell}$.

The LHC will have the potential to significantly increase the precision with which some of the electroweak observables in the fit can be measured. In particular, we expect an improvement in the determination of $m_{W}$ by a factor of $2(3)$, of $m_{t}$ by a factor of 2 (4) [118], and of $m_{h}$ by a factor of 2.5 (5) [119], at the $14 \mathrm{TeV} \mathrm{LHC,} \mathrm{with} 300 \mathrm{fb}^{-1}$ $\left(3000 \mathrm{fb}^{-1}\right)$ data. By the end of the HL-LHC's run, we expect to also have a factor of 2 improvement in the determination of $\Delta \alpha_{\text {had }}^{(5)}$ from BaBar and VEPP-2000 analyses [104]. See table 1 for a summary of current and future expected precisions. ${ }^{6}$

\footnotetext{
${ }^{6}$ We thank G. Wilson for discussions about the possibility of measuring $m_{W}$ with a precision at the level of $\sim 2 \mathrm{MeV}$ at a low energy ILC run. This improves our projected sensitivities only slightly, since the
} 


\begin{tabular}{|c|c|c|c|c|}
\hline & Present & LHC 14,300 fb & LHC 14, 3000 $\mathrm{fb}^{-1}$ & ILC (GigaZ) \\
\hline$m_{W}(\mathrm{MeV})$ & 15 & 8 & 5 & 6 \\
\hline$m_{h}(\mathrm{MeV})$ & 240 & 100 & 50 & - \\
\hline$m_{t}(\mathrm{MeV})$ & 760 & 440 & 200 & - \\
\hline$m_{Z}(\mathrm{MeV})$ & 2.1 & - & - & 1.6 \\
\hline$\Gamma_{Z}(\mathrm{MeV})$ & 2.3 & - & - & 0.8 \\
\hline$A_{b}$ & 0.02 & - & - & 0.001 \\
\hline$R_{b}^{0}\left(10^{-5}\right)$ & 69 & - & - & 14 \\
\hline$A_{\ell}\left(10^{-4}\right)$ & 18 & - & - & 1 \\
\hline
\end{tabular}

Table 1. Present and future experimental uncertainty for measurements that will be improved at the LHC and a future ILC/GigaZ. Cases where the experimental precision is not expected to significantly improve for a given observable are denoted with a dash (-). In our fits, we also assume an improvement from BaBar and VEPP-2000 data in the precision of the measurement of $\Delta \alpha_{\text {had }}^{(5)}$ to the level of $4.7 \times 10^{-5}$, compared to the present $10 \times 10^{-5}$.

The projected bound on the kinetic mixing parameter $\epsilon$ at the $14 \mathrm{TeV}$ LHC with $300 \mathrm{fb}^{-1}\left(3000 \mathrm{fb}^{-1}\right)$ data is shown as the dashed (dot-dashed) line in figure 4. This incorporates the above improvements, including $\Delta \alpha_{\text {had }}^{(5)}$. We assume progress in theoretical calculations to keep pace with improved experimental measurements, so that the approximation of neglecting theoretical uncertainties in the fit continues to be valid for these future projections. We show again two possible scenarios for the resulting limits, corresponding to two limiting assumptions about the future measured central values. Purple lines show the results of a fit assuming that the central values of all measurements remain fixed at their present values, so only the experimental uncertainties will change. Green lines show the results of a fit where the central values of all measurements are adjusted to their SM-best fit values. The mass of the $W$ boson, $m_{W}$, gives now, by far, the most important pull, followed by $A_{\ell}$ and $m_{Z}$. Figure 4 shows that we can expect an improvement of the bound on $\epsilon$ by up to $\sim 40 \%(\sim 2)$ at the at the $14 \mathrm{TeV}$ LHC with $300 \mathrm{fb}^{-1}\left(3000 \mathrm{fb}^{-1}\right)$ data. We have also verified that the bound is only weakly dependent on the correlation matrix in eq. (3.3): assuming a completely uncorrelated set of measurements would change the bound by at most a few percent.

Beyond the LHC, a possible high-luminosity and low-energy run of ILC/GigaZ ${ }^{7}$ would lead to a much more precise measurement of many precision observables [121]. In particular, measurements of the weak left-right asymmetry $A_{\ell}$ are expected to reach a precision of $10^{-4}$, reducing the current uncertainty on this observable by more than an order of magnitude. ${ }^{8}$

left-right asymmetry $A_{\ell}$ is the main pull of the fit.

${ }^{7}$ For another discussion on improved new physics reach through EWPO at future lepton colliders, see [120], which focuses on natural supersymmetry scenarios.

${ }^{8}$ This allows $\sin ^{2} \theta_{\text {eff }}^{\ell}$ to be determined with a precision of $\sim 10^{-5}$, improving the world average measurement by roughly one order of magnitude as well. However, the improvements in our limit projections derives from the increased precision on $A_{\ell}$, since $\sin ^{2} \theta_{\text {eff }}$ is not included in the fit. 
Furthermore, ILC/GigaZ will have unprecedented b-tagging capabilities. This will result in improved measurements of the left-right asymmetry, $A_{b}$, as well as $R_{b}^{0}=\Gamma(Z \rightarrow \bar{b} b) / \Gamma(Z \rightarrow$ hadrons), by a factor of 20 and 5 , respectively.

In figure 4 (dotted curves), we show the bound on $\epsilon$ using the expected uncertainties on the electroweak observables at ILC/GigaZ as shown in the latter column of table 1. For this projection, we also assume the $14 \mathrm{TeV}$ LHC $\left(3000 \mathrm{fb}^{-1}\right.$ data $)$ precision measurements of $m_{h}$ and $m_{t}$, although the bound is very similar if we take their current measured values. As seen in the figure, the sensitivity on $\epsilon$ can be increased by up to an additional factor

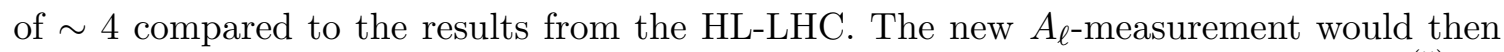
have the potential to provide the main pull in the fit, followed by $m_{Z}, m_{W}$, and $\Delta \alpha_{\text {had }}^{(5)}$.

This improvement in the indirect bound on dark photons is notable, and has the great virtue that it does not depend on how the $Z_{D}$ decays. However, in the minimal model, where the $Z_{D}$ only has SM decays available, the reach from EWPTs is not competitive with hadron colliders due to their enormous integrated luminosities, as we will now discuss.

\section{Constraining the hypercharge portal with $h \rightarrow Z Z_{D}$ decays}

In this section, we estimate the potential sensitivity of the LHC and a $100 \mathrm{TeV} p p$ collider to the exotic Higgs decay $h \rightarrow Z^{(*)} Z_{D} \rightarrow 4 \ell$. This decay mode was previously examined in [67], which recast LHC Run 1 searches for $h \rightarrow Z Z^{*} \rightarrow 4 \ell[122,123]$ to set limits on $\epsilon$ for $m_{Z_{D}} \gtrsim 10 \mathrm{GeV}$. While the resulting constraints at the level of $\epsilon \lesssim 0.04$ were weaker than indirect limits from EWPO's, the demonstrated sensitivity motivates a dedicated study of the future reach at hadron colliders. Crucially, as we also discuss in the next section, measurements of both $h \rightarrow Z_{D} Z^{(*)}$ and direct DY production of $Z_{D}$ are necessary to differentiate a kinetically mixed dark photon from a $Z^{\prime}$ with very weak gauge coupling.

The sizeable $Z_{D}$ branching ratio to leptons makes it feasible to examine a broad range of dark photon masses, including the regime where the intermediate $Z$ is off-shell. ${ }^{9}$ When $m_{h}>m_{Z}+m_{Z_{D}}$, the initial Higgs decay is two-body; when $m_{h}<m_{Z}+m_{Z_{D}}$, the initial Higgs decay is three-body. The branching ratio for this decay is shown in figure 3 (top panel) for several values of $\epsilon$. At low $m_{Z_{D}}$, where the $Z_{D}$ may be produced on shell, the dependence of $\operatorname{Br}\left(h \rightarrow Z Z_{D}\right)$ on $m_{Z_{D}}$ arises because of the mass-dependence of the $Z-Z_{D}$ mixing angle, $\cos \alpha$, see eq. (2.7). At higher masses the suppression from three-body phase space is evident.

We impose the following baseline acceptance cuts, which are modeled after [124] and similar to those in [125]:

- All electrons must satisfy $p_{T, e}>5 \mathrm{GeV}$ and $\left|\eta_{e}\right|<2.5$.

- All muons must satisfy $p_{T, \mu}>7 \mathrm{GeV}$ and $\left|\eta_{\mu}\right|<2.4$.

- Any opposite-sign, same-flavor (OSSF) lepton pair must have $m_{\ell \ell}>4 \mathrm{GeV}$.

- All events must contain exactly four accepted leptons forming two OSSF pairs, with the hardest two leptons satisfying $p_{T, 1}>20 \mathrm{GeV}$ and $p_{T, 2}>10 \mathrm{GeV}$.

\footnotetext{
${ }^{9}$ Processes with an off-shell $Z_{D}$ are higher order in $\epsilon$, and are negligible for $\epsilon \lesssim \mathcal{O}\left(10^{-2}\right)$.
} 
- The total invariant mass of the four leptons must lie in the range $120 \mathrm{GeV}<m_{4 \ell}<$ $130 \mathrm{GeV}$.

Our baseline modeling of lepton efficiencies and resolutions is based on the 7 and $8 \mathrm{TeV} \mathrm{SM} h \rightarrow 4 \ell$ searches. In particular, we employ the $p_{T^{-}}$and $\eta$-dependent electron efficiencies reported in $[122,126]$, yielding an average electron efficiency of 0.87 in SM $h \rightarrow Z Z^{*} \rightarrow 4 \ell$ events, while the muon efficiency is set to a flat 0.96. This simulation is validated against the expected numbers of signal events at 7 and $8 \mathrm{TeV}$ in [122]. As the performance achieved by the LHC experiments in LHC Run I are often quoted as goals for future performance, we will use these numbers as our benchmark scenario. In section 7 , we will also comment on the effect of varying assumed lepton $p_{T}$ thresholds, $\eta$ acceptance, and mass resolution on collider reach.

The lepton efficiency above incorporates the probability for a lepton to be insufficiently separated from a jet to pass isolation requirements in SM Higgs-like events. In [122, 126], leptons are not allowed to spoil each other's isolation requirements, and for $h \rightarrow 4 \ell$ events with SM-like kinematics, the fraction of events with a nearly collinear pair of leptons is negligibly small. We impose an additional, explicit requirement that leptons must have a minimum angular separation: $\min \left(\Delta R_{e, e}\right)>0.02$ for electrons at both 14 and $100 \mathrm{TeV}$ colliders, while for muons $\min \left(\Delta R_{\mu, \mu}\right)>0.05$ at $14 \mathrm{TeV}$ [127] and $\min \left(\Delta R_{\mu, \mu}\right)>0.02$ at $100 \mathrm{TeV}$. This requirement has negligible impact on the $h \rightarrow Z Z_{D}$ decays considered in this section, but will become important for the $h \rightarrow Z_{D} Z_{D}$ decays considered in section 6 . We consider the effect of smearing lepton energies using a Gaussian distribution with energy-dependent variance as reported in [122]. Provided that windows for cuts on lepton invariant masses are set to reasonable values (see eq. (4.2) below), we find that incorporating smearing changes our limits by only $\mathcal{O}(2 \%)$. For simplicity we neglect smearing henceforth.

We use our dark photon MadGraph model (see appendix C) to simulate gluon fusion Higgs production in MadGraph 5 and shower events in Pythia 6 [128, 129]. The inclusive Higgs production cross-section is normalized to the SM prediction of $\sigma_{g g F}=50.35 \mathrm{pb}$ at $14 \mathrm{TeV}$ and $\sigma_{g g F}=740.3 \mathrm{pb}$ at $100 \mathrm{TeV}$ [130]. The $\mathrm{SM}$ value for $\operatorname{Br}\left(h \rightarrow Z Z^{*} \rightarrow 4 \ell\right)$ is taken to be $1.26 \times 10^{-4}[131]$.

Since the reach for $h \rightarrow Z_{D} Z^{(*)} \rightarrow 4 \ell$ depends sensitively on the Higgs $p_{T}$ through lepton acceptance, we must have good control over the Higgs $p_{T}$ spectrum. This is especially a concern at $100 \mathrm{TeV}$, where many more Higgses are produced in the high- $p_{T}$ tail where the validity of the effective field theory description of $g g \rightarrow h$, used by MadGraph, breaks down. We cross-checked the Higgs $p_{T}$ spectrum from matched MadGraph/Pythia events with the $p_{T}$ spectrum predicted at NNLL+NLO by HqT $2.0[132,133]$. The two spectra are compared in figure 5, and are in good agreement for the bulk of the distribution. We reweight events to realize the NNLL+NLO $p_{T}$ spectrum , but this only gives a fractional change in sensitivity to $\epsilon$ of less than a percent.

For the SM di- $Z^{(*)} / \gamma^{*}$ background we use MadGraph to generate tree-level events, which we normalize using a K-factor of 1.2 , as computed for events with $m_{4 \ell} \in$ $(120,140) \mathrm{GeV}$ using MCFM 6.8 [134] (at both 14 and $100 \mathrm{TeV}$ ). Following [135], we multiply the diboson background by a factor of 1.5 to account for reducible backgrounds containing fake isolated leptons, notably $Z+$ jets and $t \bar{t}$. 

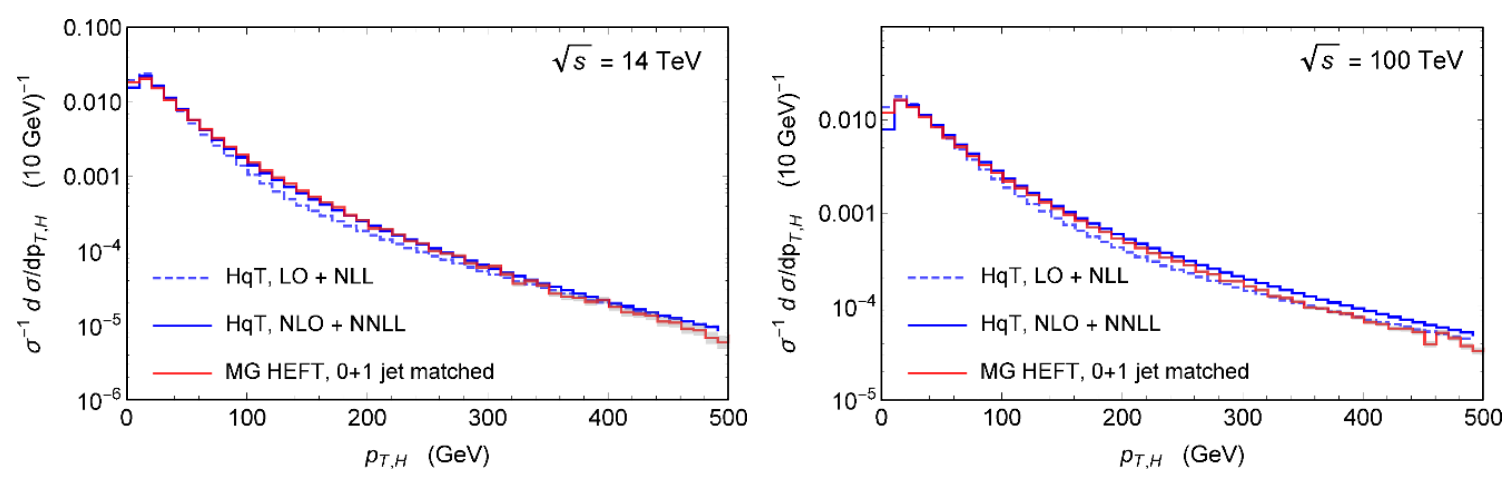

Figure 5. Predicted Higgs $p_{T}$ spectra in gluon fusion production, as calculated by matched MadGraph $5+$ Pythia 6 (red) and HqT at LO+NLL (dashed blue) and NLO+NNLL (solid blue), for $\sqrt{s}=100 \mathrm{TeV}$ (left) and $14 \mathrm{TeV}$ (right).

We define two different search regions depending on $m_{Z_{D}}$. First, we consider the case where $m_{Z_{D}}<m_{h}-m_{Z}$, and consequently the two-body decay $h \rightarrow Z Z_{D}$ can proceed onshell. We designate $M_{1}$ as the invariant mass of the pair of OSSF leptons that minimizes $\left|m_{\ell \ell}-m_{Z}\right|$, and $M_{2}$ as the invariant mass of the remaining OSSF pair. Following the SM Higgs analyses, we require

$$
M_{1,2}>12 \mathrm{GeV}
$$

to suppress backgrounds from quarkonia, and concentrate on regions not already probed by BaBar in an $e^{+} e^{-} \rightarrow \gamma Z_{D}$ search [61]. We then perform a simple bump hunt in $M_{2}$, requiring

$$
\left|M_{2}-m_{Z_{D}}\right|<\left\{\begin{array}{cc}
0.02 M_{2} & \text { (electrons) } \\
2.5\left(0.026 \mathrm{GeV}+0.013 M_{2}\right) & \text { (muons) }
\end{array}\right.
$$

These mass windows are based on current CMS energy resolutions [85], and are relatively conservative. In particular, the muon mass window we use is based on the mass resolution for forward muons, $\eta_{\mu}>0.9$, and is an underestimate of experimental capabilities.

Second, we consider the case where $m_{Z_{D}}>m_{h}-m_{Z}$. In this case the three-body process $h \rightarrow Z_{D} \ell \ell$ gives the leading contribution to the reach. Here we consider all possible divisions of events into two OSSF lepton pairs, and require that no lepton pair satisfies either $m_{\ell \ell}<12 \mathrm{GeV}$ or $\left|m_{\ell \ell}-m_{Z}\right|<15 \mathrm{GeV}$. Events are then selected if at least one OSSF lepton pair lies within

$$
\left|m_{\ell \ell}-m_{Z_{D}}\right|<M_{\text {cut }},
$$

where the mass window $M_{\text {cut }}$ depends on the flavor and mass of the lepton pair as in eq. (4.2).

Figure 6 shows our expected 95\% CLs exclusions for both the LHC and a $100 \mathrm{TeV}$ collider. These limits treat the signal mass bin as a single Poisson counting experiment, and neglect systematic uncertainties ${ }^{10}$ This limit should be compared to the limit obtained by recasting the Run I analysis for $h \rightarrow Z Z^{*} \rightarrow 4 \ell$ (shaded blue region in the figure) [67].

\footnotetext{
${ }^{10} \mathrm{~A} 10 \%$ upward shift in the background leads to a $2.3 \%$ upward shift in the exclusion reach.
} 

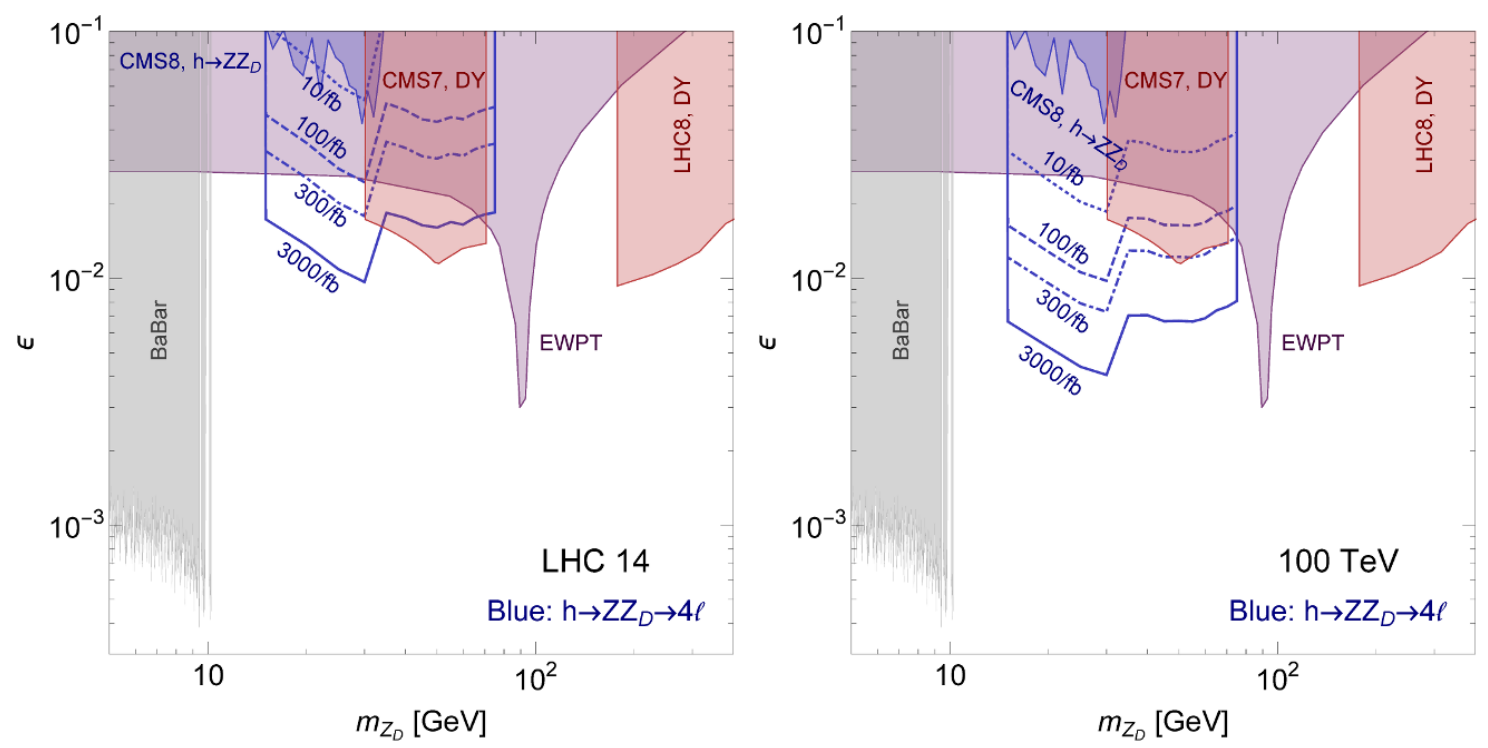

Figure 6. The blue lines show expected 95\% CLs limits on $\epsilon$ from $h \rightarrow Z_{D} Z^{(*)} \rightarrow 4 \ell$, at the LHC14 (left) and a $100 \mathrm{TeV}$ pp collider (right). Limits shown correspond to integrated luminosities of 10 (dotted), 100 (dashed), 300 (dot-dashed), and $3000 \mathrm{fb}^{-1}$ (solid) in both plots. A recast [67] of a CMS8 analysis [122] sensitive to $h \rightarrow Z Z_{D}$ is shown in the blue shaded region. The purple region shows the current EWPT constraints (this work, see section 3), while the gray region is a limit from BaBar [61]. The red regions are the bounds from Drell-Yan production of $Z_{D}[71,72,136,137]$ and are discussed in section 5 .
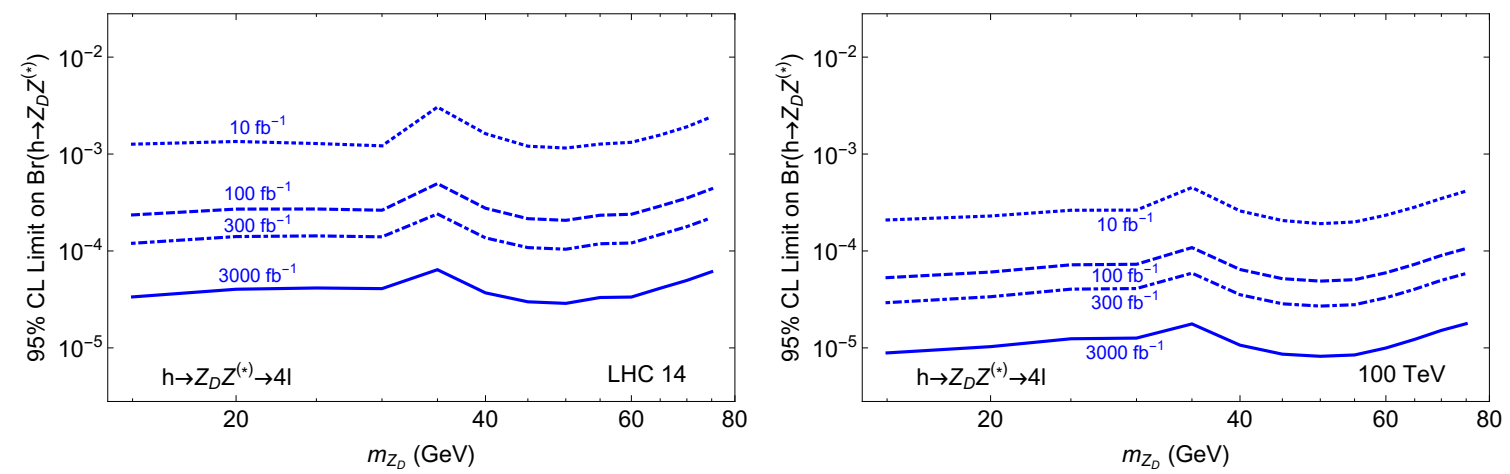

Figure 7. Projected limits on total exotic Higgs branching ratio $\operatorname{Br}\left(h \rightarrow Z_{D} Z^{*}\right)$ from the $h \rightarrow$ $Z_{D} Z^{(*)} \rightarrow 4 \ell$ search at $14 \mathrm{TeV}$ (left) and $100 \mathrm{TeV}$ (right).

It is also instructive to unfold the leptonic branching ratios of $Z_{D}$ and the possibly off-shell $Z^{(*)}$, and derive a limit on the total exotic branching fraction $\operatorname{Br}\left(h \rightarrow Z_{D} Z^{(*)}\right)$. The achievable sensitivities, shown in figure 7 , are $\sim($ few $\times) 10^{-5}$ at a $100 \mathrm{TeV}$ collider (the HL-LHC). Since both signal and main background for this search come from Higgs decays, the sensitivity achievable depends mostly on the number of Higgs bosons produced. Therefore, since the number of Higgses produced at the HL-LHC (100 TeV collider) is 2 (3) orders of magnitude higher than at any of the future proposed lepton colliders, we expect this projected limit on $\operatorname{Br}\left(h \rightarrow Z_{D} Z^{(*)}\right)$ to be at least an order of magnitude more sensitive than anything achievable at a lepton machine. 
Our HL-LHC results are less optimistic than those obtained in ref. [70] using a matrix-element-based likelihood discriminant, but we have checked that this is due almost entirely to our use of finite mass resolution in reconstructing the $Z_{D}$; in other words, the $Z_{D}$ mass peak contains almost all of the information that is useful in the statistics-limited discovery regime.

\section{Constraining the hypercharge portal with Drell-Yan $Z_{D}$ production}

The hypercharge portal coupling allows the $Z_{D}$ to be singly produced in the $s$-channel via Drell-Yan (DY) production. This gives rise to dilepton signals $p p \rightarrow Z_{D} \rightarrow \ell^{+} \ell^{-}$that show up in DY dilepton spectrum measurements, or high-mass $Z^{\prime}$ searches of the LHC experimental collaborations.

The sensitivity of DY measurements to a $Z_{D}$ below the $Z$ mass with $10 \mathrm{GeV}<m_{Z_{D}}<80 \mathrm{GeV}$ was recently explored by [72]. They recast the DY measurement at the $7 \mathrm{TeV}$ LHC [136] as a $m_{Z_{D}}$-dependent limit on $\epsilon$, and give projections for the sensitivity achievable with optimized analyses at LHC Run $1\left(8 \mathrm{TeV}, 20 \mathrm{fb}^{-1}\right)$ and at the HL-LHC $\left(14 \mathrm{TeV}, 3000 \mathrm{fb}^{-1}\right)$. The sensitivity of $Z^{\prime}$ searches for heavier dark photons $\left(m_{Z_{D}}>m_{Z}\right)$ has been explored most recently in [71] (see also [138] for an earlier study), where limits were derived on $\epsilon$ in the range $200 \lesssim m_{Z_{D}} \lesssim 2800 \mathrm{GeV}$ from published ATLAS $20 \mathrm{fb}^{-1}$ Run 1 results [137]. LEP and future lepton colliders are less sensitive to this channel than the LHC [41, 72].

In this section, we estimate how these expected constraints on $\epsilon$ from [71, 72] change at the HL-LHC (above the $Z$ mass) and at $\sqrt{s}=100 \mathrm{TeV}$ (above and below $Z$ mass). Rather than repeating the analyses of $[71,72]$, we can estimate the improved reach by taking into account the change in signal and background cross sections.

For on-shell $Z_{D}$ production, the number of expected new physics events scales with $\epsilon^{2}$. In the high-statistics limit, a 95\% CL exclusion in some signal bin is derived by solving

$$
\frac{S_{1} \epsilon^{2}}{\sqrt{B_{1}}}=c
$$

for $\epsilon$, where $\epsilon^{2} S_{1}\left(B_{1}\right)$ is the number of signal (background) events for a given search, and $c$ is some constant. The resulting limit on $\epsilon$ is

$$
\epsilon_{1}^{95 \% \mathrm{CL}}=\left(\frac{c^{2} B_{1}}{S_{1}^{2}}\right)^{1 / 4} .
$$

Suppose that we want now to rescale this $\epsilon$ limit for a different integrated luminosity and center of mass energy $\sqrt{s}$. If we know the ratio by which the signal and background number of expected events changes,

$$
B_{2}=r_{B 21} B_{1}, \quad S_{2}=r_{S 21} S_{1},
$$

we can find the new expected limit on $\epsilon$ :

$$
\epsilon_{2}^{95 \% \mathrm{CL}}=\epsilon_{1}^{95 \% \mathrm{CL}}\left(r_{B 21}\right)^{1 / 4}\left(r_{S 21}\right)^{-1 / 2} .
$$




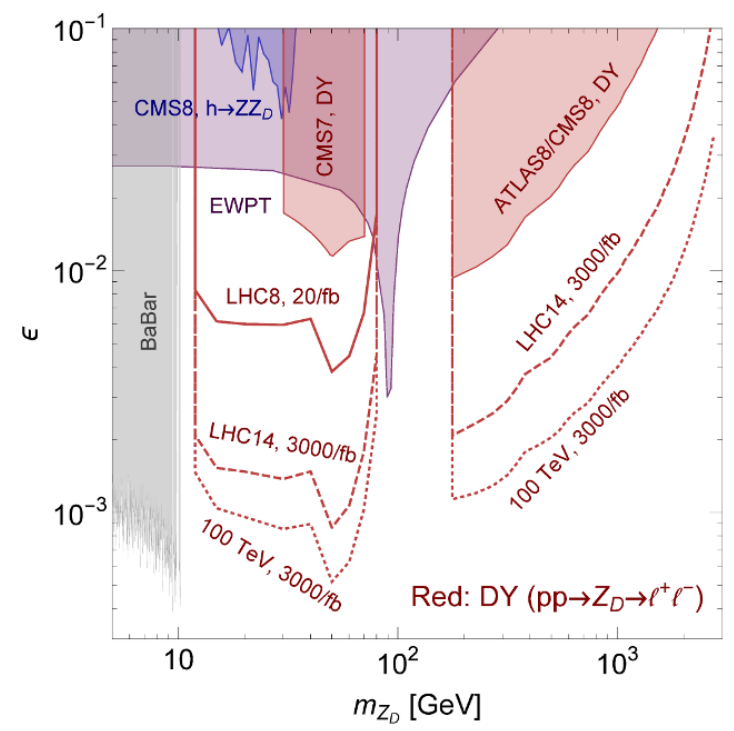

Figure 8. Prospects for $Z_{D}$ searches from DY production (red lines) at LHC8 (20 $\mathrm{fb}^{-1}$, solid), LHC14 (3000 fb $\mathrm{fb}^{-1}$, dashed), and a $100 \mathrm{TeV} p p$ collider $\left(3000 \mathrm{fb}^{-1}\right.$, dotted), with limits from existing recasts shown in shaded red (from $[71,72,136,137]$ and our rescalings, see text for details). A recast [67] of a CMS8 analysis [122] sensitive to $h \rightarrow Z Z_{D}$ is shown in the blue shaded region. The purple region shows the current EWPT constraints (this work, see Section 3), while the gray region is a limit from BaBar [61].

For the HL-LHC and a $100 \mathrm{TeV}$ collider, the $m_{\ell \ell}\left(m_{Z_{D}}\right)$ dependent rescalings $r_{B 21}\left(r_{S 21}\right)$ are easily estimated by computing the differential DY cross section $d \sigma_{\mathrm{DY}} / d m_{\ell \ell}$ (signal cross section $\sigma_{p p \rightarrow Z_{D}}$ ) at different $\sqrt{s}$ in MadGraph at LO parton level. Setting $m_{\ell \ell}=m_{Z_{D}}$, we obtain the rescaled $\epsilon$ limits shown in figure 8 .

The figure shows that DY production can be sensitive to $\epsilon \gtrsim 9 \times 10^{-4}\left(4 \times 10^{-4}\right)$ at the HL-LHC (100 TeV pp collider) While this is superior to indirect constraints from EWPTs, it does rely on the $Z_{D}$ decaying directly to SM particles. Direct DY production is also more powerful than $h \rightarrow Z Z_{D}$ searches, ${ }^{11}$ but only by a factor of a few in $\epsilon$. Crucially, a discovery in the DY channel only would be unable to distinguish between a kinetically mixed dark photon, and a new $Z^{\prime}$ which mediates a $\mathrm{U}(1)$ gauge interaction with tiny coupling constant. As we have shown in Section 4, the former scenario would leave comparable traces in the $h \rightarrow Z_{D} Z^{(*)}$ channel, while the latter scenario can only generate $h \rightarrow Z_{D} Z^{(*)}$ (i.e. $Z^{\prime} Z^{(*)}$ ) decays via fermion loops, leading to a much suppressed signal. The best-case scenario is, therefore, discovery of $Z_{D}$ in both DY and $h \rightarrow Z_{D} Z^{(*)}$ channels, allowing a precise experimental determination of the dark photon's properties.

We close this section by pointing out that publicly available DY data does not yield any constraints on $\epsilon$ in the range $90 \mathrm{GeV} \lesssim m_{Z_{D}} \lesssim 180 \mathrm{GeV}$. The minimal dark photon model provides strong experimental motivation for dedicated dark photon searches close to the $Z$-peak.

\footnotetext{
${ }^{11}$ Improvements of the dilepton mass resolution will not change this conclusion [70].
} 


\section{Constraining the Higgs and hypercharge portals with $h \rightarrow Z_{D} Z_{D}$ de- cays}

In this section, we estimate the potential sensitivity of the LHC and a $100 \mathrm{TeV} p p$ collider to the exotic Higgs decay $h \rightarrow Z_{D} Z_{D} \rightarrow 4 \ell$ (see also [93]). As for $h \rightarrow Z_{D} Z^{(*)}$, recasts of LHC Run 1 data [122, 123, 139] were used in [67] to set limits on this channel. This decay is interesting for two reasons. Firstly, for a heavy singlet scalar but non-negligible singletHiggs mixing, it is by far the most sensitive test of the $\mathrm{U}(1)_{D}$ hidden sector if $\epsilon \lesssim \mathcal{O}\left(10^{-3}\right)$. Secondly, while production of the $Z_{D}$ pair occurs through the Higgs portal, $Z_{D}$ decay has to proceed through the hypercharge portal. Therefore, observation of $h \rightarrow Z_{D} Z_{D} \rightarrow 4 \ell$ equires a non-zero value for $\epsilon$. As we describe below, the implicit $\epsilon$ sensitivity is very impressive.

\subsection{Constraining the Higgs portal from prompt $Z_{D}$ decay}

We consider a broad range of masses for $Z_{D}: 2 m_{\mu}<m_{Z_{D}}<m_{h} / 2$. Both $Z_{D}$ have to be produced on-shell, as otherwise the Higgs decay will be suppressed by $\epsilon^{2}$, and thus unobservable.

The collider analysis proceeds in large part identically to the $h \rightarrow Z_{D} Z^{(*)}$ case outlined in section 4 . The same kinematic cuts on lepton $p_{T}, \eta, \Delta R$, and $m_{4 \ell}$ are applied, and the detailed signal and background simulation is identical, including the Higgs $p_{T}$ reweighing, except that now we generate the signal process $h \rightarrow Z_{D} Z_{D} \rightarrow 4 \ell$. Recall that our analysis enforces minimum lepton separation of only $\min \left(\Delta R_{\mu \mu}\right)=0.05(0.02)$ and $\min \left(\Delta R_{e e}\right)=0.02(0.02)$ at the LHC (100 TeV collider), and is therefore sensitive to lepton jets. The new features in the $h \rightarrow Z_{D} Z_{D}$ analysis are (1) the dilepton invariant mass cuts, and (2) how the final background estimate is obtained. We divide the analysis into two regimes: the "heavy $Z_{D}$ " case with $10 \mathrm{GeV}<m_{Z_{D}}<m_{h} / 2$, and the "light $Z_{D}$ " case with $2 m_{\mu}<m_{Z_{D}}<10 \mathrm{GeV}$.

For the heavy $Z_{D}$ analysis we divide events into three families according to the flavor composition of the four lepton final state: $4 e, 4 \mu$, or $2 e 2 \mu . Z_{D}$ candidates are reconstructed by combining opposite-sign same-flavor dilepton pairs. In the cases of $4 e$ and $4 \mu$, the combinatoric uncertainty of this reconstruction is largely reduced by choosing the pairings that minimize $\left|m_{\ell \ell_{1}}-m_{\ell \ell_{2}}\right|$. The search is conducted separately for each $m_{Z_{D}}$, requiring both lepton pairs in the event to satisfy $\left|m_{\ell \ell}-m_{Z_{D}}\right|<M_{\text {cut }}$, with the same mass windows as eq. (4.2).

This double-dilepton-mass cut is extremely effective at eliminating background, to the point where simulating statistically accurate background samples in the respective signal regions is very challenging. To circumvent this issue one can make use of the fact that the background distribution in the $\left(m_{\ell \ell_{1}}, m_{\ell \ell_{2}}\right)$ plane is quite smooth, with events that pass the double-dilepton-mass cut being dominated by coincidental mispairings. This allows us to estimate the background event expectation in the small signal regions by interpolation. We construct the $\left(m_{\ell \ell_{1}}, m_{\ell \ell_{2}}\right)$ distribution for each background process (and each flavor composition of the four leptons) with large $5 \times 5 \mathrm{GeV}$ bins that each contain sufficient Monte Carlo events. The signal region is given by a small $\left(\Delta m_{1} \times \Delta m_{2}\right)$ rectangular region along the diagonal centered on $\left(m_{Z_{D}}, m_{Z_{D}}\right)$, where $\Delta m_{1,2}=2 M_{\text {cut }}$ are the total widths 
of the mass windows for each lepton pair. Rescaling the contents of the large $5 \times 5 \mathrm{GeV}$ bin along the diagonal by $\Delta m_{1} \Delta m_{2} /(5 \mathrm{GeV})^{2}$ therefore gives a suitable estimate of the background in the small signal bin.

For each flavor channel, separate signal and background expectations are obtained. 95\% CLs exclusions are obtained for both the LHC and a $100 \mathrm{TeV}$ collider, treating each flavor bin and the combined bin as single Poisson counting experiments, and selecting the best limit obtained from the $4 e, 4 \mu, 2 e 2 \mu$ or combined channel for each $m_{Z_{D}}$.

The light $Z_{D}\left(m_{Z D}<10 \mathrm{GeV}\right)$ case motivates a lepton jet analysis. Since we do not enforce lepton isolation in the reconstruction, we perform our analysis identically to the heavy case, with three exceptions. Firstly, we only use the $4 \mu$ channel, since dielectron reconstruction at such low masses is more challenging, though it might allow the search to be extended to even lower masses. Secondly, we find that at these low dilepton masses, the $m_{4 \ell}$ cut and the double-dilepton-mass cut are so restrictive that background can be neglected completely, making the limits signal-only statistically limited, though signal efficiency is limited by angular detector resolution at very low masses. We therefore set our limit at 3.8 signal events. Thirdly, for $m_{Z_{D}}$ near the $J / \Psi, \Psi(2 S)$, and $\Upsilon$ thresholds, quarkonium background is difficult to estimate and possibly large. We mark those regions with gray bands in our limit plots (taken from [100]).

The projected limits obtained for both the low and high mass $Z_{D}$ case are shown in figure 9. Also shown are limits from $8 \mathrm{TeV}$ LHC data, which supersede earlier limits [8385, 140]: a CMS search for $h \rightarrow 2 a \rightarrow 4 \mu$ [86], where we assume that efficiencies for pseudoscalar and dark vector decay to di-muon jets are the same, and recasts by [67] of the CMS $h \rightarrow Z Z^{*} \rightarrow 4 \ell$ search [122] and the ATLAS $Z Z$ cross section measurement [139]. It will be possible to improve on these present limits with only $10 \mathrm{fb}^{-1}$ of $14 \mathrm{TeV}$ data. The HL-LHC probes $\operatorname{Br}\left(h \rightarrow Z_{D} Z_{D}\right) \gtrsim 10^{-6}$, while a $100 \mathrm{TeV}$ collider will this sensitivity by more than an order of magnitude. Since this search is signal limited, we expect this projected bound to be 2 to 3 orders of magnitude better than anything achievable by one of the proposed lepton colliders.

The dimensionless parameter $\kappa^{\prime}$ that determines the exotic branching fraction depends on both the Higgs portal coupling and the singlet scalar mass, see eq. (2.34). Constraints for $\kappa^{\prime}$ are also shown in figure 9 (bottom). HL-LHC measurements could probe this parameter at the few $10^{-5}$ level. A $100 \mathrm{TeV}$ collider could push the sensitivity by almost another order of magnitude. The results of our study demonstrate the remarkable power of leptonic searches to set bounds on the mixing between the Higgs and an additional scalar. The sensitivity lies many orders of magnitude beyond the (indirect) sensitivity to non-SM decays from Higgs coupling measurements.

As mentioned in section 2.2, one could also imagine probing the Higgs portal by making use of direct dark Higgs production, which proceeds through its inherited SM couplings and occurs at the same order in $\kappa$ as $h \rightarrow Z_{D} Z_{D}$. For small enough $m_{Z_{D}}$ the dark Higgs would decay to two dark photons, giving a similar signal to the process studied in this section. However, we have checked that the exotic Higgs decay $h \rightarrow Z_{D} Z_{D} \rightarrow 4 \ell$ provides the best sensitivity to $\kappa^{\prime}$ if $h \rightarrow s s$ is kinematically forbidden, as we assume throughout this paper. For $m_{s}>m_{h} / 2$, gluon fusion is the dominant production mode for the dark 

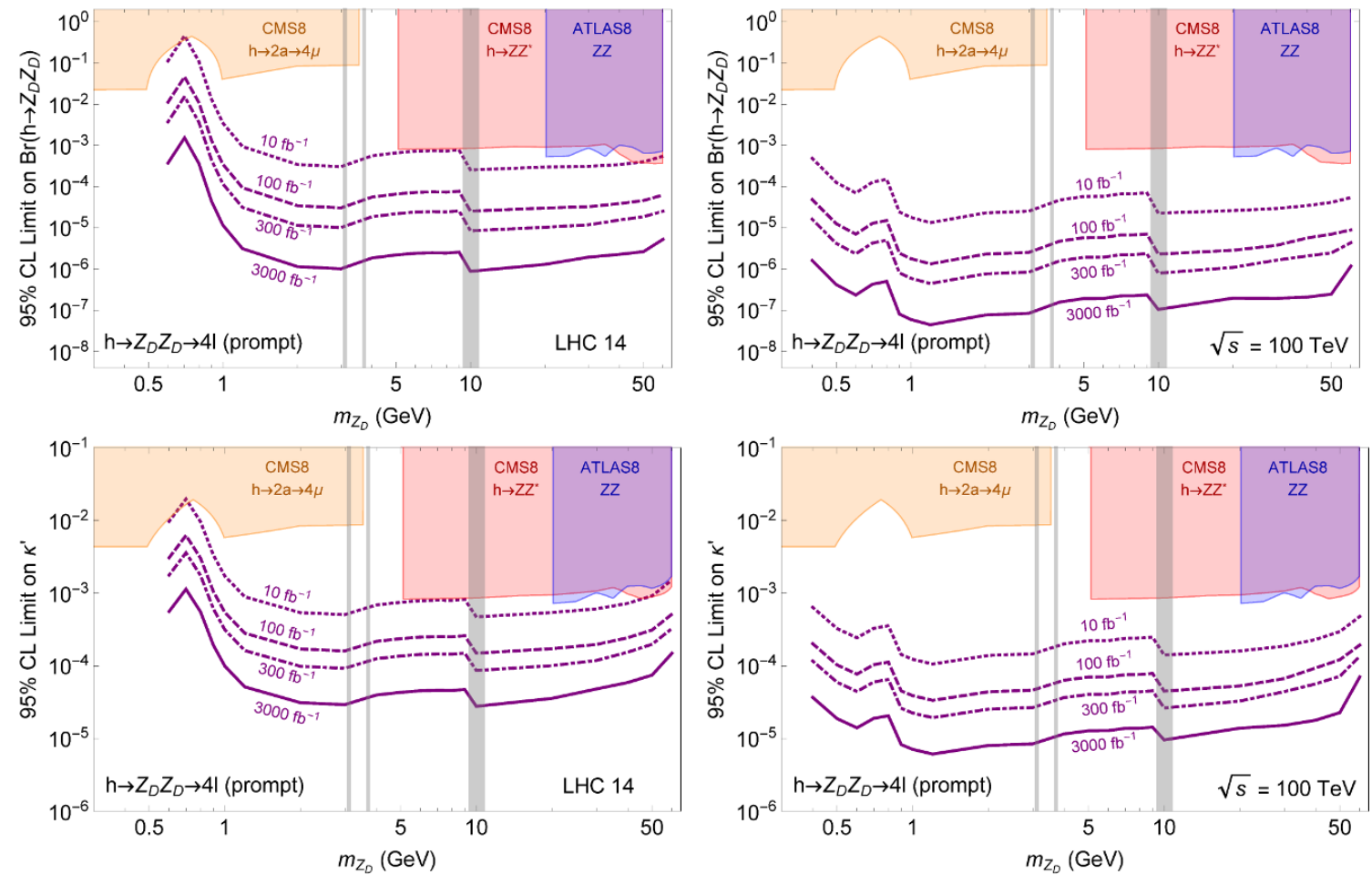

Figure 9. Expected 95\% CLs limits on the total exotic Higgs decay branching ratio, $\operatorname{Br}\left(h \rightarrow Z_{D} Z_{D}\right)$ (top), and the effective Higgs mixing parameter $\kappa^{\prime}$ (bottom) at the LHC (left) and a $100 \mathrm{TeV}$ pp collider (right). Gray bands correspond to regions where quarkonium background may invalidate our analysis. The limits obtained in [67] from a recast of LHC Run 1 results are shown in red $\left(h \rightarrow Z Z^{*} \rightarrow 4 \ell\right.$ search by CMS [122]) and blue (ATLAS $Z Z$ cross section measurement [139]) shaded regions. The limit from the $\mathrm{CMS} 8 \mathrm{TeV} h \rightarrow 2 a \rightarrow 4 \mu$ search [86] is shaded in orange, assuming the efficiencies for pseudoscalar and dark photon decay to muons are the same.

scalar. Due to the small width of the SM Higgs,

$$
\sigma(g g \rightarrow h) \times B R\left(h \rightarrow Z_{D} Z_{D}\right) \gg \sigma(g g \rightarrow s) \times B R\left(s \rightarrow Z_{D} Z_{D}\right)
$$

even for $m_{h} / 2<m_{s}<m_{h}$. Since the $h \rightarrow Z_{D} Z_{D}$ search has very low background it provides the best sensitivity to the Higgs portal coupling $\kappa$.

\subsection{Constraints on kinetic mixing from displaced $Z_{D}$ decays}

The $\operatorname{Br}\left(h \rightarrow Z_{D} Z_{D}\right)$ limits of figure 9 assume that all the $Z_{D}$ decay promptly. As shown in figure 2 , this requires $\epsilon \gtrsim 10^{-5}-10^{-3}$ over the $2 m_{\mu}<m_{Z_{D}}<m_{h} / 2$ mass range. Therefore, discovery of this exotic Higgs decay can also give sensitivity to much smaller values of $\epsilon$ than any channel that relies on $\epsilon$ for production, e.g. the direct DY production and $h \rightarrow Z_{D} Z^{(*)}$ decays considered in sections 4 and 5 . By considering macroscopic decay lengths of the $Z_{D}$, we can extend our sensitivity to even smaller values of $\epsilon$, giving an even more impressive sensitivity to the hypercharge portal. For example, extending the above analysis to include displaced $Z_{D}$ decay to leptons with a decay length of up to $\sim 10 \mathrm{~cm}$ gives sensitivity to $\epsilon \sim 10^{-8}-10^{-6}$, assuming a signal reconstruction efficiency similar to prompt $Z_{D}$ decays. 
In fact, a recent ATLAS analysis [141] has set such limits on displaced dark photons in a supersymmetrized version of the model considered here. As we demonstrate below, similar analyses are highly motivated for the minimal dark photon model.

Let us assume for simplicity that a displaced dilepton pair search has the same reconstruction efficiency for all $Z_{D}$ decays within some length $L$ of the interaction point as for prompt decays. ${ }^{12}$ In that case, the effective visible exotic Higgs branching fraction to four leptons is given by

$$
\operatorname{Br}_{\text {eff }}=\operatorname{Br}\left(h \rightarrow Z_{D} Z_{D}\right) \operatorname{Br}\left(Z_{D} \rightarrow \ell \ell\right)^{2} P\left(L, \sqrt{s}, m_{Z_{D}}, \epsilon\right),
$$

where $P\left(L, \sqrt{s}, m_{Z_{D}}, \epsilon\right)$ is the probability that both $Z_{D}$ decay before traveling a length $L$ :

$$
P\left(L, \sqrt{s}, m_{Z_{D}}, \epsilon\right)=\int d b_{1} d b_{2} f\left(\sqrt{s}, m_{Z_{D}} ; b_{1}, b_{2}\right)\left[1-e^{-L /\left(b_{1} \lambda\right)}\right]\left[1-e^{-L /\left(b_{2} \lambda\right)}\right] .
$$

Here $\lambda=\lambda\left(m_{Z_{D}}, \epsilon\right)=c / \Gamma_{Z_{D}}\left(m_{Z_{D}}, \epsilon\right)$ is the proper decay length of the dark photon, shown in figure $2, b_{i}=\left|\vec{p}_{Z_{D i}}\right| / m_{Z_{D}}$ are the boost factors of each $Z_{D}$ in the event, and $f\left(\sqrt{s}, m_{Z_{D}} ; b_{1}, b_{2}\right)$ is the probability distribution for an event to have boost factors $\left(b_{1}, b_{2}\right)$ for the two $Z_{D}$ s.

For the purposes of this estimate we can take our limits on $\operatorname{Br}\left(h \rightarrow Z_{D} Z_{D} \rightarrow 4 \ell\right)$ in figure 9 to be limits on $\mathrm{Br}_{\text {eff }}$ in eq. (6.2). We now estimate the ability of this search to constrain $\epsilon$ for different values of $\operatorname{Br}\left(h \rightarrow Z_{D} Z_{D}\right)$ and reasonable choices of $L$, to demonstrate the reach that might be achieved at HL-LHC or a future $100 \mathrm{TeV}$ collider.

The joint distribution of boosts $f\left(\sqrt{s}, m_{Z_{D}} ; b_{1}, b_{2}\right)$ can be obtained from signal Monte Carlo events, but evaluating eq. (6.3) in the $\left(m_{Z_{D}}, \epsilon\right)$ plane is quite computationally expensive, and neither necessary nor instructive for our estimate. We can simplify eq. (6.3) by first making use of the fact that the two boost factors $b_{1}$ and $b_{2}$ are highly correlated and tend to be similar in each event. Letting $f\left(\sqrt{s}, m_{Z_{D}} ; b\right)$ be the boost factor distribution of a single $Z_{D}$ in the signal event sample, $P$ can be approximated as

$$
P\left(L, \sqrt{s}, m_{Z_{D}}, \epsilon\right) \approx \int d b f\left(\sqrt{s}, m_{Z_{D}} ; b\right)\left[1-e^{-L /(b \lambda)}\right]^{2} .
$$

The most important behavior of $P$ can be captured by the two limits

$$
P\left(L, \sqrt{s}, m_{Z_{D}}, \epsilon\right) \approx \begin{cases}1 & \text { for } L \gg b \lambda \\ \left(\frac{L}{b \lambda}\right)^{2} & \text { for } L \ll b \lambda\end{cases}
$$

where $b$ is a representative boost factor in the event kinematics for a given $m_{Z_{D}}$. Expanding eq. (6.4) for small $L$,

$$
\begin{aligned}
P\left(L, \sqrt{s}, m_{Z_{D}}, \epsilon\right) & \approx \int d b f\left(\sqrt{s}, m_{Z_{D}} ; b\right)\left[\left(\frac{L}{b \lambda}\right)^{2}+\ldots\right] \\
& =\left(\frac{L}{\lambda}\right)^{2} \int d b \frac{f\left(\sqrt{s}, m_{Z_{D}} ; b\right)}{b^{2}}+\ldots
\end{aligned}
$$

\footnotetext{
${ }^{12} \mathrm{~A}$ displaced lepton search will have lower background but probably also lower signal efficiency, so this is a crude estimate, but it is sufficient to illustrate our point.
} 

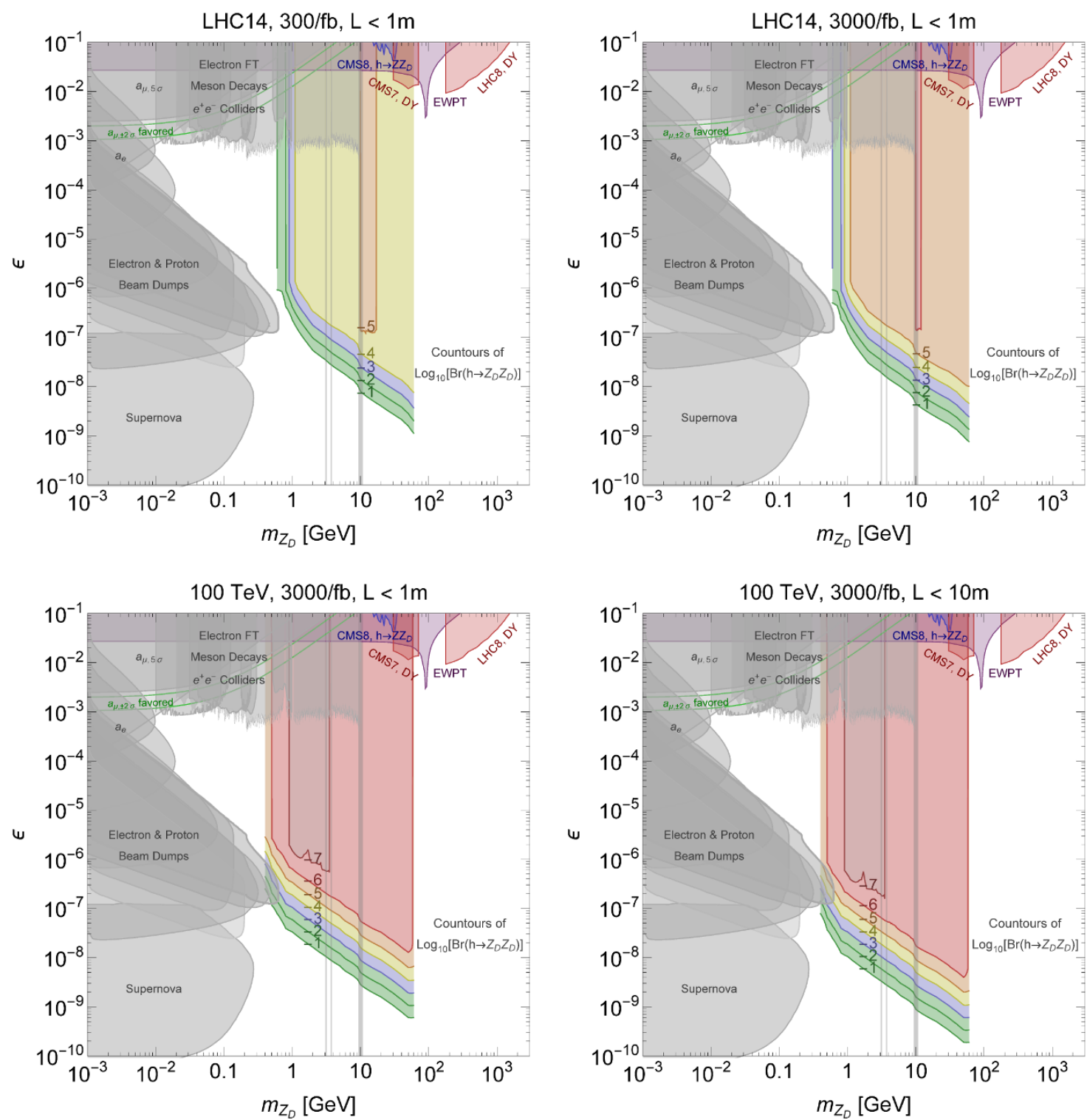

Figure 10. Estimate of expected $95 \%$ CLs limits on $\epsilon$ for different $\operatorname{Br}\left(h \rightarrow Z_{D} Z_{D}\right)$ at the LHC (top left), HL-LHC (top right), and a $100 \mathrm{TeV}$ collider (bottom), assuming a displaced lepton jet search has the same sensitivity to decays within the given distance from the interaction point as a prompt $Z_{D} Z_{D}$ search (see figure 9). A detector size $L$ of $1 \mathrm{~m}$ is assumed for all plots except for the bottom right plot, which assumes $10 \mathrm{~m}$ for the $100 \mathrm{TeV}$ collider. Gray shaded regions show current constraints (see section 1 for references). 


$$
=\left(\frac{L}{\tilde{b} \lambda}\right)^{2}+\ldots,
$$

where we have defined an 'effective average boost factor'

$$
\tilde{b}=\tilde{b}\left(m_{Z_{D}}, \sqrt{s}\right) \equiv\left[\int d b \frac{f\left(\sqrt{s}, m_{Z_{D}} ; b\right)}{b^{2}}\right]^{-1 / 2} .
$$

We can then write

$$
P\left(L, \sqrt{s}, m_{Z_{D}}, \epsilon\right) \approx\left[1-e^{-L /(\tilde{b} \lambda)}\right]^{2},
$$

which gives the correct limit, eq. (6.5), for small $L$. The $m_{Z_{D}}$ dependence of $\tilde{b}$ is nearly identical for $\sqrt{s}=100$ and $14 \mathrm{TeV}$, since the $Z_{D}$ kinematics are dominated by the decay of a Higgs particle produced mostly near threshold. In fact, assuming both $Z_{D}$ come from the decay of a stationary Higgs gives

$$
b=\sqrt{\frac{m_{h}^{2}}{4 m_{Z_{D}}^{2}}-1},
$$

which is a very good approximation for $\tilde{b}$ everywhere except the near threshold region where $m_{Z_{D}} \approx m_{h} / 2$.

Using eq. (6.8), it is straightforward to convert limits on $\mathrm{Br}_{\text {eff }}$ (eq. (6.2)) from figure 9 to $\epsilon$ limits as a function of $m_{Z_{D}}$ for different $\operatorname{Br}\left(h \rightarrow Z_{D} Z_{D}\right)$. This is shown in figure 10 for the LHC and HL-LHC, assuming displaced vertices out to $1 \mathrm{~m}$ from the interaction point can be reconstructed, as well as for a $100 \mathrm{TeV}$ collider with $3000 \mathrm{fb}^{-1}$, assuming displaced vertex reconstruction out to either 1 or $10 \mathrm{~m}$.

A $10 \%$ invisible Higgs branching ratio to two long-lived $Z_{D}$ is not presently excluded [142, 143], and even future lepton colliders like ILC and TLEP would only constrain such an invisible decay at the $0.5 \%$ level $[119,144]$. For such relatively large $\operatorname{Br}\left(h \rightarrow Z_{D} Z_{D}\right)$, the HL-LHC $(100 \mathrm{TeV}$ collider $)$ offers sensitivity to $\epsilon \gtrsim 10^{-9}-10^{-6}$ $\left(10^{-10}-10^{-7}\right)$. This is is many orders of magnitude beyond anything achievable with searches that rely on the hypercharge portal for $Z_{D}$ production. Even a very small Higgs portal can allow us to glimpse deeply into the dark sector.

\section{$7 \quad$ Impact of future detector design}

Detector capabilities are important for assessing the detailed reach of $p p$ colliders for both decays $h \rightarrow Z Z_{D} \rightarrow 4 \ell$ and $h \rightarrow Z_{D} Z_{D} \rightarrow 4 \ell$. Our forecasts are based on LHC8 lepton performance, which may differ in several aspects from the ultimate detector performance at a $100 \mathrm{TeV}$ collider. To illustrate the importance of detector design on the reach, we examine in this section how our expected limits depend on key assumptions about lepton identification and reconstruction.

A future detector could perform worse than an LHC8 detector with regards to lepton reconstruction $p_{T}$ thresholds. In the analyses of sections 4 and 6 , we implicitly assumed $100 \%$ trigger efficiency for $p_{L 1}^{T}>20 \mathrm{GeV}$ and $p_{L 2}^{T}>10 \mathrm{GeV}$. As we show in figure 11 (left), 

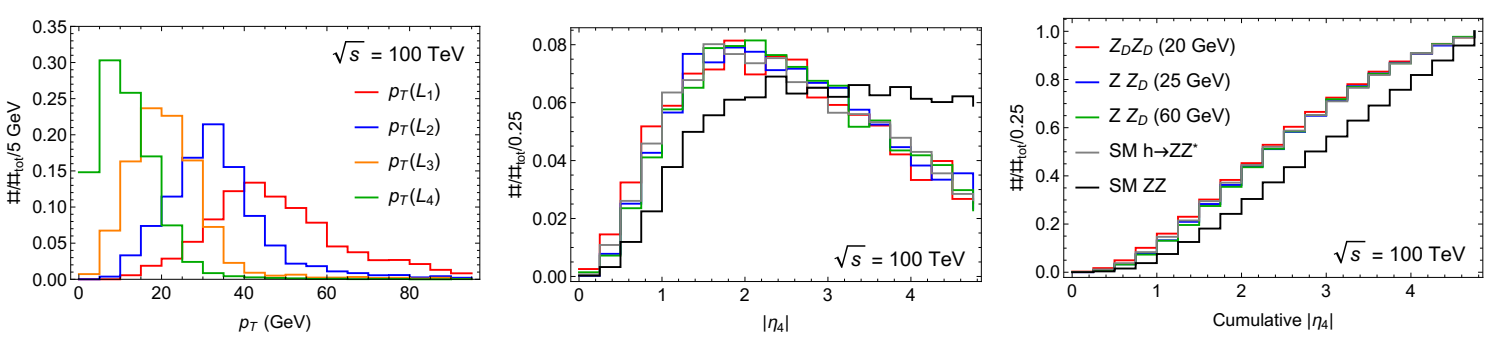

Figure 11. Left: distribution of $p_{L 1,2,3,4}^{T}$ for $h \rightarrow Z_{D} Z_{D} \rightarrow 4 \ell$ with $m_{Z_{D}}=20 \mathrm{GeV}$. Middle and right: $\left|\eta_{4}\right|$ and cumulative $\left|\eta_{4}\right|$ distribution for $h \rightarrow Z_{D} Z_{D} \rightarrow 4 \ell\left(m_{Z_{D}}=20 \mathrm{GeV}\right)$, $h \rightarrow Z Z_{D} \rightarrow 4 \ell\left(m_{Z_{D}}=25,60 \mathrm{GeV}\right)$ and SM backgrounds $h \rightarrow Z Z^{*}, Z Z$. The only applied cut is $120<m_{4 \ell}<130 \mathrm{GeV}$.

these thresholds can be raised by 10 or even $20 \mathrm{GeV}$ (for $\ell_{2}$ ) with relatively little loss of signal acceptance. More serious is the dependence on the minimum lepton reconstruction threshold, which was assumed to be $p_{T}>7,5 \mathrm{GeV}$ for electrons and muons respectively. Raising this threshold to $10 \mathrm{GeV}$ degrades signal efficiency by about $50 \%$ in both $h \rightarrow$ $Z Z_{D} \rightarrow 4 \ell$ and $h \rightarrow Z_{D} Z_{D} \rightarrow 4 \ell$ analyses, with a $\sim 20 \%$ loss of $\epsilon$ and $\kappa^{\prime}$ sensitivity. This is shown in figure 12 for a $100 \mathrm{TeV}$ collider.

On the other hand, a future detector could perform better than an LHC8 detector with regards to rapidity acceptance and mass resolution. Signal acceptance can be notably improved if lepton coverage is extended to higher values of $|\eta|$. Figure 11 (middle and right) shows the distribution of the maximum $|\eta|$ among the four leptons in both SM and BSM $h \rightarrow 4 \ell$ events, along with the distribution in the main background $Z^{(*)} Z^{(*)}$, before any $p_{T}$ requirements are imposed. Note that, for both SM and BSM Higgs bosons, requiring all four leptons to satisfy $|\eta|<2.5$ eliminates approximately half of the signal events. We therefore investigate the possibility of raising the maximum rapidity to 4 . Conversely, background rejection could be improved by improving the dilepton mass resolution. We consider the change in reach if it is possible to employ an optimistic mass window of

$$
\left|M_{\ell \ell}-m_{Z_{D}}\right|<0.015 M_{\ell \ell}
$$

for both electrons and muons. The impact of these two possible improvemenets on $\epsilon$ and $\kappa^{\prime}$ sensitivity for a $100 \mathrm{TeV}$ collider is shown in figure 13 .

Extending lepton $\eta$ coverage by itself does not necessarily improve the sensitivity to $h \rightarrow Z^{(*)} Z_{D}$, as acceptance for the main background, SM $h \rightarrow Z Z^{*} \rightarrow 4 \ell$, increases as well. This is especially notable for $m_{Z_{D}}>m_{h}-m_{Z}$, where SM background is nonnegligible. In this region, extending lepton $\eta$ coverage is actually detrimental without other improvements, as can be seen from the dotted line in figure 13 (left). The importance of reducing SM background can be seen from the dashed line, which shows the improvement in sensitivity given the improvement in lepton mass resolution according to eq. (7.1). Notably, combining the improvement in mass resolution with extended $\eta$ coverage yields a $\gtrsim 20 \%$ improvement in the reach in $\epsilon$ (see dot-dashed line in the figure). We also consider the possibility of increasing the rapidity coverage at the HL-LHC [145]. For $h \rightarrow Z Z_{D}$, this 

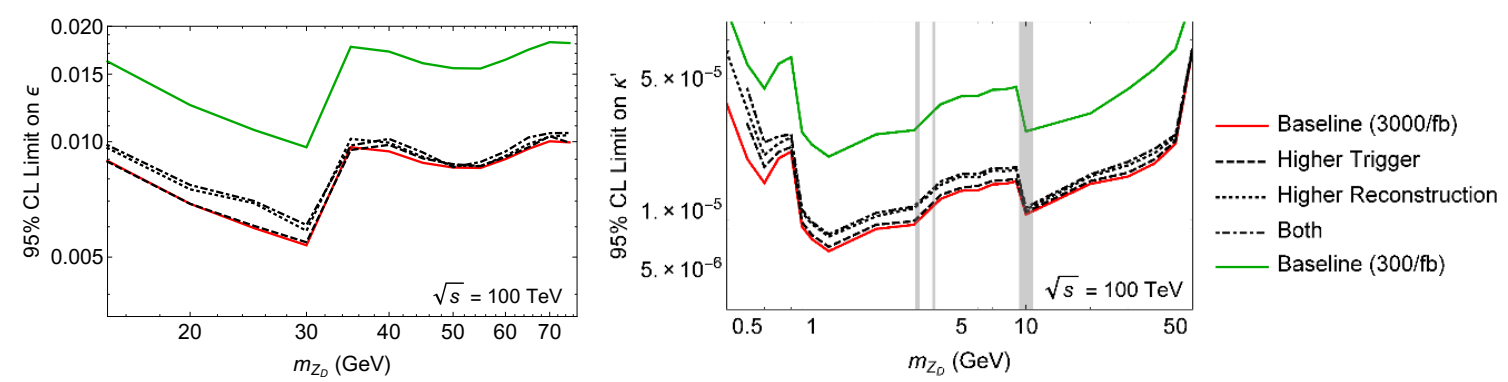

Figure 12. Estimated 95\% CLs sensitivity to $\epsilon$ from $h \rightarrow Z Z_{D} \rightarrow 4 \ell$ (left) and $\kappa^{\prime}$ from $h \rightarrow$ $Z_{D} Z_{D} \rightarrow 4 \ell$ (right) at a $100 \mathrm{TeV}$ collider with $3000 \mathrm{fb}^{-1}$. Baseline selection criteria in red, raising trigger thresholds $p_{L_{1,2}}^{T}$ from $(20,10) \mathrm{GeV}$ to $(30,20) \mathrm{GeV}$ gives the dashed line, raising lepton reconstruction thresholds $p_{T}^{e, \mu}$ from $(7,5) \mathrm{GeV}$ to $10 \mathrm{GeV}$ gives the dotted line, dot-dashed shows both. The projected $300 \mathrm{fb}^{-1}$ baseline limit is shown for scale in green.
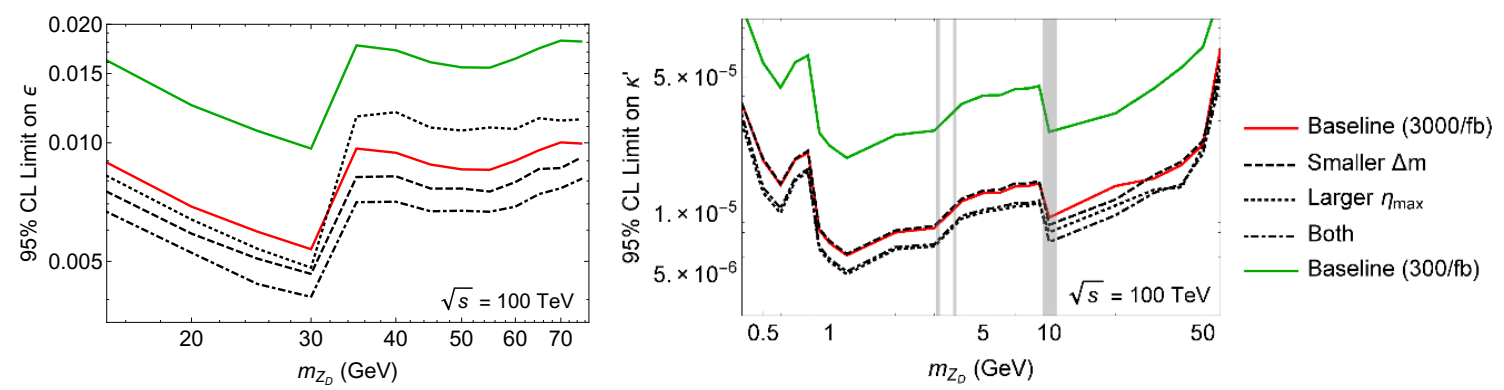

Figure 13. Estimated 95\% CLs sensitivity to $\epsilon$ from $h \rightarrow Z Z_{D} \rightarrow 4 \ell$ (left) and $\kappa^{\prime}$ from $h \rightarrow$ $Z_{D} Z_{D} \rightarrow 4 \ell$ (right) at a $100 \mathrm{TeV}$ collider with $3000 \mathrm{fb}^{-1}$. Baseline selection criteria in red, improved mass resolution (according to eq. (7.1)) in the dashed line, increased lepton acceptance $(|\eta|<4)$ in the dotted line, dot-dashed shows both. The projected $300 \mathrm{fb}^{-1}$ baseline limit is shown for scale in green.

improves signal acceptance by $\approx 25 \%$; however without an improvement in mass resolution over our existing projections, we find it does not help to improve limits on $h \rightarrow Z_{D} \ell \ell \rightarrow 4 \ell$.

The situation is simpler for $h \rightarrow Z_{D} Z_{D}$. Due to the double-dilepton-mass cut, backgrounds are so low that the increased signal acceptance for larger $\eta$ coverage more than offsets the elevated background levels. The improved mass window by itself slightly improves higher-mass limits on $\kappa^{\prime}$ (where there is some background) but has no effect on the background-free low-mass limits. Best results are achieved by utilizing both improvements, which increases $\kappa^{\prime}$ sensitivity by about $25 \%$.

\section{Discussion and conclusions}

Dark sectors with a broken $\mathrm{U}(1)_{D}$ gauge group that kinetically mixes with the SM hypercharge are well motivated and appear in a variety of new physics scenarios. In this paper, we showed that high-energy proton-proton and electron-positron colliders, like the LHC14, a $100 \mathrm{TeV}$ collider, and an ILC/GigaZ, have excellent sensitivity to dark photons. In fact, they may provide the only probe for dark photons with masses above $10 \mathrm{GeV}$, as 


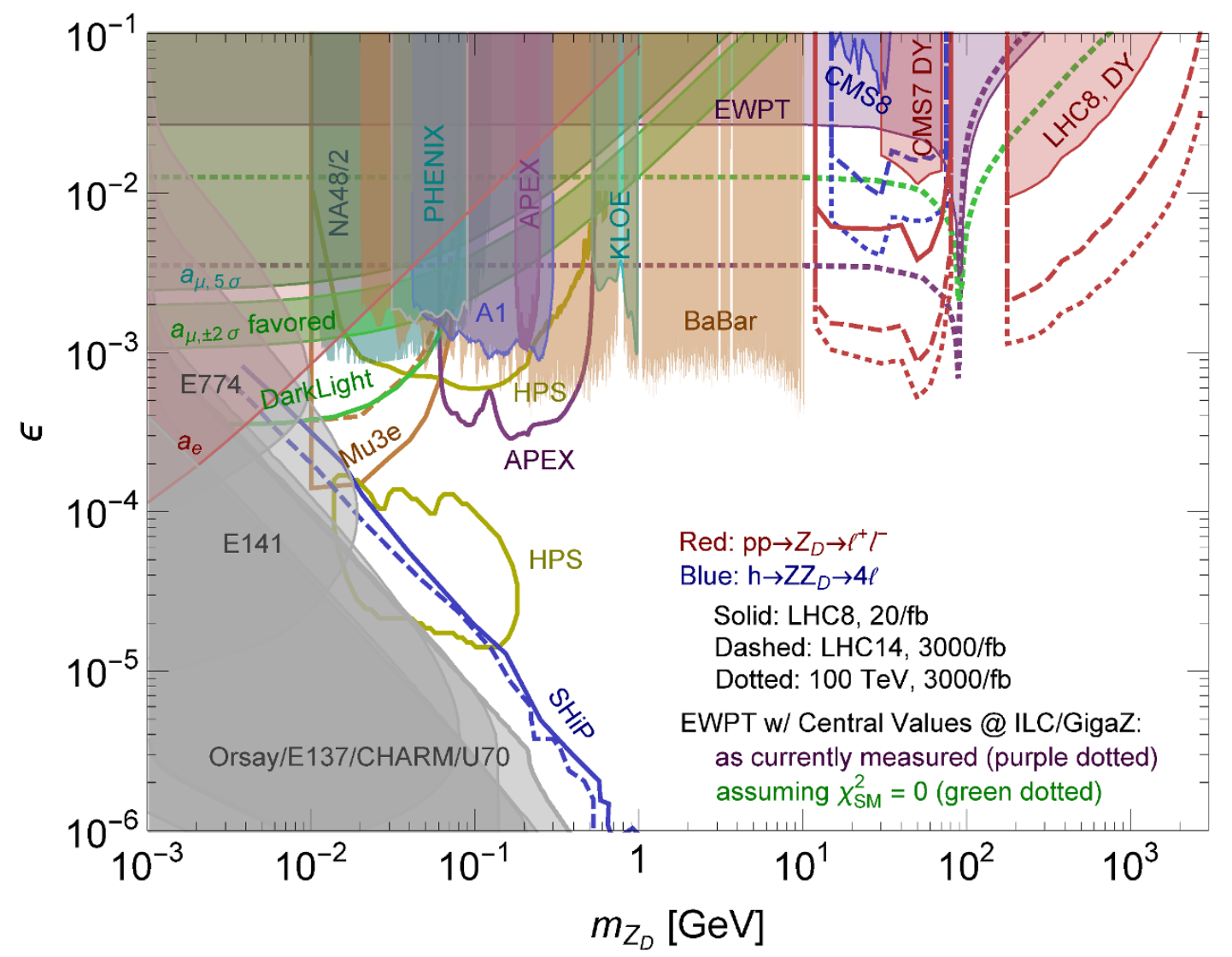

Figure 14. Summary of dark photon constraints and prospects (see section 1 for references). High-energy colliders (LHC14, $100 \mathrm{TeV}$, ILC/GigaZ) are uniquely sensitive to dark photons with $m_{Z_{D}} \gtrsim 10 \mathrm{GeV}$, while precision QED observables and searches at $B$ - and $\Phi$-factories, beam dump experiments, and fixed target-experiments probe lower masses. Dark photons can be detected at high-energy colliders in a significant part of open parameter space in the exotic decay of the $125 \mathrm{GeV}$ Higgs boson, $h \rightarrow Z Z_{D} \rightarrow 4 \ell$, (blue curves) in Drell-Yan events, $p p \rightarrow Z_{D} \rightarrow \ell \ell$, (red curves) and through improved measurements of electroweak precision observables (green/purple dashed curves). Note that all constraints and prospects assume that the dark photon decays directly to SM particles, except for the precision measurements of the electron/muon anomalous magnetic moment and the electroweak observables. If, in addition to kinetic mixing, the $125 \mathrm{GeV}$ Higgs mixes with the dark Higgs that breaks the dark $\mathrm{U}(1)$, then the decay $h \rightarrow Z_{D} Z_{D}$ would set constraints on $\epsilon$ that are orders of magnitude more powerful than other searches down to dark photon masses of $\sim 100 \mathrm{MeV}$, see figure 10 .

high-intensity beam-dump experiments or $B$-factories do not have enough energy to probe this mass region. Moreover, the $125 \mathrm{GeV}$ Higgs boson plays a pivotal role in these searches, providing additional motivation to search for its possible exotic (non-standard) decays.

If the only connection between the dark and SM sectors is kinetic mixing (i.e. the the hypercharge portal), then the dark photon can be produced in Drell-Yan events and in the exotic Higgs decay $h \rightarrow Z_{D} Z^{(*)}$. In addition, it would change the SM expectation for electroweak precision observables. A renormalizable mixing between the $125 \mathrm{GeV}$ Higgs and 
the hidden-sector Higgs (i.e. a Higgs portal) is expected to be present if the dark photon mass is generated by a Higgs mechanism. This would allow for the exotic Higgs decay $h \rightarrow Z_{D} Z_{D}$. We have investigated these various possibilities, summarizing them in figure 14 for the pure hypercharge portal case and in figure 10 if there is an additional Higgs mixing.

Our conclusions are the following:

- Drell-Yan production is the most promising discovery channel of dark photons if the dark Higgs does not mix with SM-like Higgs boson (see figure 8 and [71, 72]). Recasts of existing LHC Run 1 data already set some of the best limits for some ranges of dark photon masses above $10 \mathrm{GeV}$, and especially for masses above about $180 \mathrm{GeV}$. Data from the upcoming HL-LHC run and a potential future $100 \mathrm{TeV}$ collider can probe $\epsilon \gtrsim$ $9 \times 10^{-4}$ and $4 \times 10^{-4}$, approaching the same sensitivity to dark photon masses above $10 \mathrm{GeV}$ that BaBar data achieved below $10 \mathrm{GeV}$. Additional experimental analyses of the DY dilepton mass spectrum near the $Z$-peak are motivated to help fill in the gap between the high- and low-mass DY bounds.

- Exotic Higgs decays $h \rightarrow Z_{D} Z^{(*)} \rightarrow 4 \ell$ provide an additional powerful probe of dark photons with masses below the $Z$-boson (see figure 7 ), and serve as complementary discovery channels to DY production. Moreover, a discovery in the Drell-Yan channel alone would not be sufficient to pinpoint the properties of a new vector boson; the sizeable branching ratio predicted for $h \rightarrow Z_{D} Z^{(*)}$ in the case of a kinetically-mixed $Z_{D}$ makes this exotic Higgs decay a key diagnostic in establishing the properties of any newly discovered vector boson.

- Electroweak precision constraints have the distinctive advantage of being independent of the dark photon decay mode (see figure 4). Existing constraints require $\epsilon \lesssim 3 \times 10^{-2}$ for masses below $\sim 80 \mathrm{GeV}$. The upcoming HL-LHC can probe $\epsilon$ down to almost $10^{-2}$, while an ILC/GigaZ can probe down to almost $3 \times 10^{-3}$ in the same mass range. Above the $Z$-pole, the constraint and prospects weaken, but are stronger than any other existing constraint up to about $180 \mathrm{GeV}$. If the dark photon decays directly to SM particles, the above-mentioned searches in DY events and exotic Higgs decays will be significantly more powerful in the entire mass range above $10 \mathrm{GeV}$ than measurements of electroweak observables.

- For $\epsilon \lesssim 10^{-3}$, direct production of the dark photon through the hypercharge portal is very unlikely at current or future planned colliders. However, if the dark Higgs mixes with the $125 \mathrm{GeV}$ Higgs, the spectacular exotic decay $h \rightarrow Z_{D} Z_{D} \rightarrow 4 \ell$ gives an additional probe into the hidden sector through the Higgs portal. The effective Higgs mixing parameter, see eq. (2.34), can be constrained by the HL-LHC $(100 \mathrm{TeV}$ collider) to be $\kappa^{\prime} \lesssim$ few $\times 10^{-5}$ (few $\times 10^{-6}$ ), see figure 9 . Since the detection of the $h \rightarrow Z_{D} Z_{D} \rightarrow 4 \ell$ decay relies on the dark photon decaying directly to leptons, any such discovery would also yield sensitivity to the hypercharge portal at the $\epsilon \lesssim$ $10^{-7}-10^{-6}$ level, which is the smallest kinetic mixing for which almost all dark photons decay inside of the detector. 
- Future lepton colliders will constrain the invisible Higgs decay branching fraction at the $0.5 \%$ level $[119,144]$. If $\operatorname{Br}\left(h \rightarrow Z_{D} Z_{D}\right)$ is of this size, then then $\epsilon$ values as low $10^{-9}-10^{-6}\left(10^{-10}-10^{-7}\right)$ can be probed at the HL-LHC $(100 \mathrm{TeV}$ collider $)$ by looking for highly displaced dark photon decays, see figure 10.

Our forecasts for the sensitivity of exotic Higgs decays are based on LHC8 lepton performance, which may differ in several aspects from the ultimate detector performance at a $100 \mathrm{TeV}$ collider. We have investigated the sensitivity of our conclusions to varying those assumptions, and find that the plausible range of lepton $p_{T}$ thresholds, mass resolutions, and rapidity acceptances can affect the limits on $\epsilon$ and $\kappa^{\prime}$ at the $\sim 20 \%$ level. Our results can also be applied to estimate sensitivity to the rare SM $h \rightarrow 4 \ell$ decays via exclusive quarkonia decays [146-148].

Finally, we have made a fully consistent MadGraph implementation of the minimal dark photon model publicly available for future investigations, see appendix C.

This work showcases one example of the impressive sensitivity to light hidden sectors provided by future colliders. Discovery requires both large center of mass energies and enormous production rates for relatively light particles, and in particular the SM Higgs boson. Future hadron colliders will offer unique discovery avenues onto both frontiers, provided sensitivity to relatively low- $p_{T}$ objects is maintained.

\section{Acknowledgments}

We thank Wolfgang Altmannshofer, Brian Calvert, Aviana Essig, Eder Izaguirre, Michael Peskin, Kevin Pedro, and Graham W. Wilson for helpful discussions. DC is supported in part by the NSF under Grants PHY-PHY-0969739 and PHY-1315155, and by the Maryland Center for Fundamental Physics. RE is supported by the DoE Early Career research program DESC0008061 and through a Sloan Foundation Research Fellowship. JS is grateful to the Mainz Institute for Theoretical Physics for its hospitality and partial support during the completion of this work. SG would like to thank the Center for Future High Energy Physics (CFHEP) in Beijing for hospitality and partial support. Research at Perimeter Institute is supported by the Government of Canada through Industry Canada and by the Province of Ontario through the Ministry of Economic Development \& Innovation. Part of this work was completed at the Aspen Center for Physics, which operates under the NSF Grant 1066293

\section{A Tables of branching ratios and $Z_{D}$ full width}

For $m_{Z_{D}}>12 \mathrm{GeV}$, table 2 shows the dark photon total width, leptonic branching fraction, and the exotic Higgs decay branching fractions. This includes 3-loop QCD corrections using the results of [101]. In each case, $\ell$ is taken to mean both $e$ and $\mu$. $\left(Z_{D}\right.$ partial widths to each are identical in this mass range.) See section 2.1 for more details.

For $m_{Z_{D}}<12 \mathrm{GeV}$, the same information can be computed using $R(s)$ data from [100] and LO leptonic $Z_{D}$ widths from eq. (2.12) to compute the total $Z_{D}$ width via eq. (2.14). The resulting leptonic branching ratios are then given by eq. (2.15), while the exotic Higgs 


\begin{tabular}{|c|c|c|c|c|}
\hline$\frac{m_{Z_{D}}}{\mathrm{GeV}}$ & $\frac{\Gamma_{Z_{D}}}{\epsilon^{2}(\mathrm{GeV})}$ & $\operatorname{Br}\left(Z_{D} \rightarrow \ell \ell\right)$ & $\frac{\operatorname{Br}\left(h \rightarrow Z_{D} Z^{(*)} \rightarrow 4 \ell\right)}{\epsilon^{2}}$ & $\frac{\operatorname{Br}\left(h \rightarrow Z_{D} Z_{D} \rightarrow 4 \ell\right)}{\kappa^{\prime 2}}$ \\
\hline 12 & 0.217 & 0.289 & 0.00180 & 93.6 \\
\hline 14 & 0.253 & 0.288 & 0.00252 & 91.4 \\
\hline 16 & 0.290 & 0.288 & 0.00338 & 89.0 \\
\hline 18 & 0.327 & 0.287 & 0.00439 & 86.4 \\
\hline 20 & 0.365 & 0.286 & 0.00555 & 83.4 \\
\hline 22 & 0.403 & 0.285 & 0.00681 & 80.2 \\
\hline 24 & 0.442 & 0.284 & 0.00814 & 76.9 \\
\hline 26 & 0.482 & 0.283 & 0.00940 & 73.3 \\
\hline 28 & 0.522 & 0.281 & 0.0104 & 69.6 \\
\hline 30 & 0.564 & 0.280 & 0.0108 & 65.8 \\
\hline 32 & 0.607 & 0.278 & 0.00961 & 61.9 \\
\hline 34 & 0.651 & 0.275 & 0.00599 & 58.0 \\
\hline 36 & 0.697 & 0.273 & 0.00380 & 54.1 \\
\hline 38 & 0.746 & 0.270 & 0.00312 & 50.2 \\
\hline 40 & 0.797 & 0.267 & 0.00280 & 46.4 \\
\hline 42 & 0.851 & 0.263 & 0.00263 & 42.6 \\
\hline 44 & 0.909 & 0.259 & 0.00253 & 38.9 \\
\hline 46 & 0.972 & 0.254 & 0.00247 & 35.3 \\
\hline 48 & 1.04 & 0.249 & 0.00242 & 31.7 \\
\hline 50 & 1.12 & 0.244 & 0.00238 & 28.2 \\
\hline 52 & 1.20 & 0.238 & 0.00235 & 24.8 \\
\hline 54 & 1.29 & 0.231 & 0.00232 & 21.4 \\
\hline 56 & 1.40 & 0.223 & 0.00229 & 17.9 \\
\hline 58 & 1.53 & 0.215 & 0.00225 & 14.2 \\
\hline 60 & 1.68 & 0.206 & 0.00221 & 10.1 \\
\hline 62 & 1.86 & 0.196 & 0.00217 & 4.28 \\
\hline 64 & 2.09 & 0.186 & 0.00212 & - \\
\hline 66 & 2.37 & 0.174 & 0.00208 & - \\
\hline 68 & 2.73 & 0.163 & 0.00203 & - \\
\hline 70 & 3.21 & 0.150 & 0.00198 & - \\
\hline 72 & 3.87 & 0.138 & 0.00193 & - \\
\hline 74 & 4.79 & 0.125 & 0.00189 & - \\
\hline 76 & 6.17 & 0.113 & 0.00184 & - \\
\hline 78 & 8.34 & 0.102 & 0.00179 & - \\
\hline 80 & 12.0 & 0.0914 & 0.00175 & - \\
\hline 82 & 18.9 & 0.0827 & 0.00170 & - \\
\hline 84 & 34.1 & 0.0754 & 0.00165 & - \\
\hline 86 & 78.2 & 0.0695 & 0.00161 & - \\
\hline 88 & 308. & 0.0647 & 0.00156 & - \\
\hline
\end{tabular}

Table 2. Branching ratios and total widths in the dark photon model as a function of dark photon mass $m_{Z_{D}}$, for $\epsilon, \kappa^{\prime} \ll 1$. Three-loop QCD corrections are included using the results of [101]. See section 2.1 for more details. 
decay branching fractions for $h \rightarrow Z_{D} Z_{(D)} \rightarrow 4 \ell$ can then be computed analytically with eqs. (2.19) and (2.33).

The methods we employ in section 2.1 can also be applied to compute the various widths and branching ratios for $m_{Z_{D}}>m_{Z}$. However, the LO approximation for $Z_{D}$ partial widths, eq. (2.12), is an excellent approximation for such high masses. The threebody width $\Gamma\left(h \rightarrow Z_{D} Z^{(*)} \rightarrow 4 \ell\right)$ can be computed with eq. (2.20) or in MadGraph, see appendix $\mathrm{C}$.

We also make tables of all branching ratios and partial widths used in this paper, for all $m_{Z_{D}}<m_{Z}$, available for download at the Exotic Higgs Decay Working Group website and the website for the Madgraph model. See appendix $C$ for the urls.

\section{B $\quad Z_{D}$ contributions to precision electroweak observables}

In this appendix, we give more details on computing the effects of $Z_{D}$ on several of the electroweak precision observables used for our fit (see eq. (3.1), with the exception of $\left.\sin ^{2} \theta_{\text {eff }}^{\ell}\left(Q_{\mathrm{FB}}\right)\right)$.

The first set of observables, the mass of the $Z$ and of the $W$ bosons and the total width of the $W$ boson, $\Gamma_{W}$, are only affected by the shift in the $Z$ mass. In particular, the physical mass of the $Z$ boson does not correspond anymore to the input value in (3.2) but it is given by the expression in eq. (2.8) where $m_{Z, 0}$ is our input value, over which we are marginalizing. The computation of the $W$ boson mass follows closely the computation in the framework of the SM. More specifically, for the $W$ mass, we have to solve iteratively the equation

$$
m_{W}^{2}\left(1-\frac{m_{W}^{2}}{m_{Z}^{2}}\right)=\frac{\pi \alpha}{\sqrt{2} G_{F}}(1+\Delta r)
$$

where $G_{F}$ is the Fermi constant and $\alpha$ is the fine structure constant. $\Delta r$, which also depends on the $W$ mass, summarizes all radiative corrections, computed fully at the two-loop level in the Standard Model [111]. The leading NP effect in our theory comes from the shift of $Z$ mass, compared to the input value $m_{Z, 0}$ that enters (B.1). For the $W$ boson width, $\Gamma_{W}$, we employ the one loop parametrization in [112]. From there we can see that, again, the main NP effect comes from the shift in the $Z$ mass through $m_{W}$.

Next, we discuss those observables measured at the $Z$ peak that are affected both by the shift in the $Z$ mass and by the shift of the $Z$ couplings. The partial widths of the $Z$ into fermions can be expressed in terms of the effective vector- and axial-vector couplings, $v_{f}$ and $a_{f}$, of the $\mathrm{Z}$ boson to leptons at the Z-pole $i \bar{f} \gamma^{\mu}\left(v_{f}+a_{f} \gamma_{5}\right) f Z_{\mu}$, as [112]

$$
\Gamma_{f}=\frac{G_{F} m_{Z}^{3}}{6 \sqrt{2} \pi}\left[\left(\left(v_{f}^{2}+\delta_{i m \kappa}^{f}\right) C_{f V}+a_{f}^{2} C_{f A}\right]\left(1+\frac{3}{4} Q^{2} \frac{\hat{\alpha}\left(m_{Z}\right)}{\pi}\right)+\Delta_{E W / Q C D}^{f},\right.
$$

where $Q$ is the electric charge of the fermion $f$, while $C_{f V}$ and $C_{f A}$ describe corrections to the color factor in the vector and axial-vector currents, and $\Delta_{E W / Q C D}^{f}$ are mixed QED and QCD corrections taken from [149]. Finally, $\delta_{i m \kappa}^{f}$ is the correction from the imaginary part of the loop-induced mixing of the photon and the $\mathrm{Z}$ boson. In our theory, several partial widths of the $Z$ boson will be affected due to the shift in the $Z$ couplings $v_{f}, a_{f}$, 


\begin{tabular}{|c|c|c|c|}
\hline Observable & Measurement & Observable & Measurement \\
\hline$m_{Z}$ & $(91.1875 \pm 0.0021) \mathrm{GeV}$ & $A_{\ell}$ & $0.1499 \pm 0.0018$ \\
\hline$\Gamma_{Z}$ & $(2.4952 \pm 0.0023) \mathrm{GeV}$ & $A_{b}$ & $0.923 \pm 0.020$ \\
\hline$\sigma_{\text {had }}^{0}$ & $(41.540 \pm 0.037) \mathrm{nb}$ & $A_{c}$ & $0.670 \pm 0.027$ \\
\hline$R_{\ell}^{0}$ & $20.767 \pm 0.025$ & $A_{F B}^{\ell, 0}$ & $0.0171 \pm 0.0010$ \\
\hline$R_{b}^{0}$ & $0.21629 \pm 0.00066$ & $A_{F B}^{b, 0}$ & $0.0992 \pm 0.0016$ \\
\hline$R_{c}^{0}$ & $0.1721 \pm 0.0030$ & $A_{F B}^{c, 0}$ & $0.0707 \pm 0.0035$ \\
\hline$m_{W}$ & $(80.385 \pm 0.015) \mathrm{GeV}$ & $\Gamma_{W}$ & $(2.085 \pm 0.042) \mathrm{GeV}$ \\
\hline$m_{t}$ & $(173.34 \pm 0.76) \mathrm{GeV}$ & $m_{h}$ & $(125.14 \pm 0.24) \mathrm{GeV}$ \\
\hline$\Delta \alpha_{\text {had }}^{(5)}$ & $0.2757 \pm 0.0001$ & & \\
\hline
\end{tabular}

Table 3. Experimental values, as measured at LEP, SLC, Tevatron and LHC, of the several EWPOs. Note that for $A_{\ell}$ we are using an average of LEP and SLC measurements, for the top mass we are using the newest world average, combination of Tevatron and LHC results [150] and not the newest CMS result in [151].

as well as by kinematics, since $\Gamma_{f} \propto m_{Z}$ and the physical $m_{Z}$ is not given by the input value $m_{Z, 0}$, see eq. (2.5).

From these partial widths, it is easy to compute the remaining electroweak observables: the Z-peak hadronic cross-section, $\sigma_{\text {had }}^{0}$ and the partial width ratios, $R_{\ell}^{0}, R_{c}^{0}, R_{b}^{0}$ :

$$
\begin{aligned}
\sigma_{\text {had }}^{0} & =\frac{12 \pi}{m_{Z}^{2}} \frac{\Gamma_{e} \Gamma_{\text {had }}}{\Gamma_{Z}^{2}}, \\
R_{\ell}^{0} & =\frac{\Gamma_{\text {had }}}{\Gamma_{\ell}}, R_{q}^{0}=\frac{\Gamma_{q}}{\Gamma_{\text {had }}},
\end{aligned}
$$

where $q=c, b, \Gamma_{\text {had }}=\Gamma_{u}+\Gamma_{d}+\Gamma_{c}+\Gamma_{s}+\Gamma_{b}$, and $\Gamma_{Z}=\Gamma_{\text {had }}+\Gamma_{\ell}+\Gamma_{\nu}$, with $\Gamma_{\nu}$ being the partial width of the $Z$ into neutrinos.

Furthermore, the left-right asymmetry parameters $A_{\ell}, A_{c}, A_{b}$ can be expressed at the tree level by

$$
A_{f}^{\text {tree }}=\frac{2 v_{f} / a_{f}}{1+\left(v_{f} / a_{f}\right)^{2}} .
$$

To take into account higher order SM corrections, we express the lepton asymmetry parameters as functions of the effective weak mixing angles $\sin ^{2} \theta_{\mathrm{eff}}^{f}$,

$$
A_{f} \equiv \frac{2\left(1-4|Q| \sin ^{2} \theta_{\mathrm{eff}}^{f}\right)}{1+\left(1-4|Q| \sin ^{2} \theta_{\mathrm{eff}}^{f}\right)^{2}} .
$$

In the $\mathrm{SM}, \sin ^{2} \theta_{\text {eff }}^{f}$ is the solution to the equation

$$
\sin ^{2} \theta_{\mathrm{eff}}^{f}=\left(1-\frac{m_{W}^{2}}{m_{Z}^{2}}\right)\left(1+\Delta \kappa_{f}\right)
$$

where the first part takes into account the relation between the Fermi constant $G_{F}$ and the $W$ boson mass. The second part takes into account the corrections to the $Z$-fermion 
vertex form factors and it depends only weakly on the value of $m_{W}$. The SM numerical result of eq. (B.7) is expressed in terms of a fitting function that depends on the input parameters in (3.2). The fitting function of our theory, which we have to use for the leftright asymmetry parameters $A_{f}$, will be given by the SM function (with the appropriate input value $m_{Z, 0}$ ) plus tree-level corrections due to the shift of the $Z$ couplings.

Finally, the forward-backward asymmetries, $A_{F B}^{\ell,}, A_{F B}^{c, 0}, A_{F B}^{b, 0}$ are given by

$$
A_{F B}^{f, 0}=\frac{3}{4} A_{\ell} A_{f} .
$$

For completeness, we show in table 3 the collection of experimental values used in our fit.

\section{MadGraph implementation of higgsed dark photon model}

We make a fully consistent MadGraph 5 [94] implementation of the higgsed dark photon model, constructed in FeynRules 2.0 [95], publicly available. It can be found at the website of the Exotic Higgs Decay Working Group,

$$
\text { http://exotichiggs.physics.sunysb.edu/, }
$$

or directly at

$$
\text { http://insti.physics.sunysb.edu/ curtin/hahm_mg.html. }
$$

Open Access. This article is distributed under the terms of the Creative Commons Attribution License (CC-BY 4.0), which permits any use, distribution and reproduction in any medium, provided the original author(s) and source are credited.

\section{References}

[1] Y. Gershtein et al., Working group report: new particles, forces and dimensions, arXiv:1311.0299 [INSPIRE].

[2] Workshop on future high energy circular colliders webpage, http://indico.ihep.ac.cn/conferenceDisplay.py?confId $=3813$.

[3] Future circular collider study kick-off meeting webpage, https://indico.cern.ch/event/282344/.

[4] N. Zhou, D. Berge, L. Wang, D. Whiteson and T. Tait, Sensitivity of future collider facilities to WIMP pair production via effective operators and light mediators, arXiv: 1307.5327 [INSPIRE].

[5] T. Cohen et al., SUSY simplified models at 14, 33 and 100 TeV proton colliders, JHEP 04 (2014) 117 [arXiv:1311.6480] [INSPIRE].

[6] S. Jung and J.D. Wells, Gaugino physics of split supersymmetry spectra at the LHC and future proton colliders, Phys. Rev. D 89 (2014) 075004 [arXiv:1312.1802] [INSPIRE].

[7] T.G. Rizzo, Exploring new gauge bosons at a 100 TeV collider, Phys. Rev. D 89 (2014) 095022 [arXiv: 1403.5465] [INSPIRE]. 
[8] M. Low and L.-T. Wang, Neutralino dark matter at $14 \mathrm{TeV}$ and $100 \mathrm{TeV}$, JHEP 08 (2014) 161 [arXiv: 1404.0682] [INSPIRE].

[9] T. Cohen, R.T. D'Agnolo, M. Hance, H.K. Lou and J.G. Wacker, Boosting stop searches with a $100 \mathrm{TeV}$ proton collider, JHEP 11 (2014) 021 [arXiv:1406.4512] [INSPIRE].

[10] A.J. Larkoski and J. Thaler, Aspects of jets at 100 TeV, Phys. Rev. D 90 (2014) 034010 [arXiv:1406.7011] [INSPIRE].

[11] A. Hook and A. Katz, Unbroken $\mathrm{SU}(2)$ at a $100 \mathrm{TeV}$ collider, JHEP 09 (2014) 175 [arXiv: 1407.2607] [INSPIRE].

[12] S. Gori, S. Jung, L.-T. Wang and J.D. Wells, Prospects for electroweakino discovery at a $100 \mathrm{TeV}$ hadron collider, JHEP 12 (2014) 108 [arXiv:1410.6287] [INSPIRE].

[13] D. Curtin, P. Meade and C.-T. Yu, Testing electroweak baryogenesis with future colliders, JHEP 11 (2014) 127 [arXiv: 1409.0005] [INSPIRE].

[14] S. Chang, J. Galloway, M. Luty, E. Salvioni and Y. Tsai, Phenomenology of induced electroweak symmetry breaking, arXiv:1411.6023 [INSPIRE].

[15] Z. Chacko, H.-S. Goh and R. Harnik, The twin Higgs: natural electroweak breaking from mirror symmetry, Phys. Rev. Lett. 96 (2006) 231802 [hep-ph/0506256] [INSPIRE].

[16] G. Burdman, Z. Chacko, H.-S. Goh and R. Harnik, Folded supersymmetry and the LEP paradox, JHEP 02 (2007) 009 [hep-ph/0609152] [INSPIRE].

[17] N. Craig and K. Howe, Doubling down on naturalness with a supersymmetric twin Higgs, JHEP 03 (2014) 140 [arXiv:1312.1341] [INSPIRE].

[18] N. Craig, S. Knapen and P. Longhi, Neutral naturalness from the orbifold Higgs, arXiv: 1410.6808 [INSPIRE].

[19] G. Burdman, Z. Chacko, R. Harnik, L. de Lima and C.B. Verhaaren, Colorless top partners, a $125 \mathrm{GeV}$ Higgs and the limits on naturalness, arXiv:1411.3310 [INSPIRE].

[20] V. Silveira and A. Zee, Scalar phantoms, Phys. Lett. B 161 (1985) 136 [InSPIRE].

[21] M. Pospelov, A. Ritz and M.B. Voloshin, Secluded WIMP dark matter, Phys. Lett. B 662 (2008) 53 [arXiv:0711.4866] [INSPIRE].

[22] D. Feldman, B. Körs and P. Nath, Extra-weakly interacting dark matter, Phys. Rev. D 75 (2007) 023503 [hep-ph/0610133] [INSPIRE].

[23] D.E. Morrissey and M.J. Ramsey-Musolf, Electroweak baryogenesis, New J. Phys. 14 (2012) 125003 [arXiv: 1206.2942] [INSPIRE].

[24] M.J. Strassler and K.M. Zurek, Echoes of a hidden valley at hadron colliders, Phys. Lett. B 651 (2007) 374 [hep-ph/0604261] [INSPIRE].

[25] B. Holdom, Two U(1)'s and $\epsilon$ charge shifts, Phys. Lett. B 166 (1986) 196 [INSPIRE].

[26] P. Galison and A. Manohar, Two Z's or not two Z's?, Phys. Lett. B 136 (1984) 279 [INSPIRE].

[27] K.R. Dienes, C.F. Kolda and J. March-Russell, Kinetic mixing and the supersymmetric gauge hierarchy, Nucl. Phys. B 492 (1997) 104 [hep-ph/9610479] [INSPIRE].

[28] J. Jaeckel and A. Ringwald, The low-energy frontier of particle physics, Ann. Rev. Nucl. Part. Sci. 60 (2010) 405 [arXiv: 1002. 0329] [INSPIRE]. 
[29] J.L. Hewett et al., Fundamental physics at the intensity frontier, arXiv:1205.2671 [INSPIRE].

[30] R. Essig et al., Working group report: new light weakly coupled particles, arXiv:1311.0029 [INSPIRE].

[31] J.D. Bjorken, R. Essig, P. Schuster and N. Toro, New fixed-target experiments to search for dark gauge forces, Phys. Rev. D 80 (2009) 075018 [arXiv:0906.0580] [INSPIRE].

[32] B. Batell, M. Pospelov and A. Ritz, Probing a secluded U(1) at B-factories, Phys. Rev. D 79 (2009) 115008 [arXiv: 0903.0363] [INSPIRE].

[33] R. Essig, P. Schuster and N. Toro, Probing dark forces and light hidden sectors at low-energy $e^{+} e^{-}$colliders, Phys. Rev. D 80 (2009) 015003 [arXiv:0903.3941] [InSPIRE].

[34] M. Freytsis, G. Ovanesyan and J. Thaler, Dark force detection in low energy e-p collisions, JHEP 01 (2010) 111 [arXiv:0909.2862] [INSPIRE].

[35] R. Essig, P. Schuster, N. Toro and B. Wojtsekhowski, An electron fixed target experiment to search for a new vector boson $A^{\prime}$ decaying to $e^{+} e^{-}$, JHEP 02 (2011) 009

[arXiv: 1001.2557] [INSPIRE].

[36] J. Blümlein and J. Brunner, New exclusion limits for dark gauge forces from beam-dump data, Phys. Lett. B 701 (2011) 155 [arXiv:1104.2747] [INSPIRE].

[37] S. Andreas, C. Niebuhr and A. Ringwald, New limits on hidden photons from past electron beam dumps, Phys. Rev. D 86 (2012) 095019 [arXiv: 1209.6083] [InSPIRE].

[38] M. Pospelov, Secluded U(1) below the weak scale, Phys. Rev. D 80 (2009) 095002 [arXiv:0811.1030] [INSPIRE].

[39] M. Reece and L.-T. Wang, Searching for the light dark gauge boson in GeV-scale experiments, JHEP 07 (2009) 051 [arXiv:0904.1743] [INSPIRE].

[40] BABAR collaboration, B. Aubert et al., Search for dimuon decays of a light scalar boson in radiative transitions $\Upsilon \rightarrow \gamma A^{0}$, Phys. Rev. Lett. 103 (2009) 081803 [arXiv:0905.4539] [INSPIRE].

[41] A. Hook, E. Izaguirre and J.G. Wacker, Model independent bounds on kinetic mixing, Adv. High Energy Phys. 2011 (2011) 859762 [arXiv: 1006. 0973] [INSPIRE].

[42] J.D. Bjorken et al., Search for neutral metastable penetrating particles produced in the SLAC beam dump, Phys. Rev. D 38 (1988) 3375 [InSPIRE].

[43] E.M. Riordan et al., A search for short lived axions in an electron beam dump experiment, Phys. Rev. Lett. 59 (1987) 755 [INSPIRE].

[44] A. Bross et al., A search for shortlived particles produced in an electron beam dump, Phys. Rev. Lett. 67 (1991) 2942 [INSPIRE].

[45] KLOE-2 collaboration, D. Babusci et al., Limit on the production of a light vector gauge boson in phi meson decays with the KLOE detector, Phys. Lett. B 720 (2013) 111 [arXiv:1210.3927] [INSPIRE].

[46] F. Archilli et al., Search for a vector gauge boson in phi meson decays with the KLOE detector, Phys. Lett. B 706 (2012) 251 [arXiv:1110.0411] [InSPIRE].

[47] APEX collaboration, S. Abrahamyan et al., Search for a new gauge boson in electron-nucleus fixed-target scattering by the APEX experiment, Phys. Rev. Lett. 107 (2011) 191804 [arXiv:1108.2750] [INSPIRE]. 
[48] A1 collaboration, H. Merkel et al., Search for light gauge bosons of the dark sector at the Mainz microtron, Phys. Rev. Lett. 106 (2011) 251802 [arXiv:1101.4091] [INSPIRE].

[49] J.B. Dent, F. Ferrer and L.M. Krauss, Constraints on light hidden sector gauge bosons from supernova cooling, arXiv:1201.2683 [INSPIRE].

[50] H. Davoudiasl, H.-S. Lee and W.J. Marciano, Dark side of Higgs diphoton decays and muon g-2, Phys. Rev. D 86 (2012) 095009 [arXiv:1208.2973] [InSPIRE].

[51] H. Davoudiasl, H.-S. Lee and W.J. Marciano, 'Dark' Z implications for parity violation, rare meson decays and Higgs physics, Phys. Rev. D 85 (2012) 115019 [arXiv:1203.2947] [INSPIRE].

[52] H. Davoudiasl, H.-S. Lee, I. Lewis and W.J. Marciano, Higgs decays as a window into the dark sector, Phys. Rev. D 88 (2013) 015022 [arXiv:1304.4935] [INSPIRE].

[53] M. Endo, K. Hamaguchi and G. Mishima, Constraints on hidden photon models from electron g-2 and hydrogen spectroscopy, Phys. Rev. D 86 (2012) 095029 [arXiv:1209. 2558] [INSPIRE].

[54] J. Balewski et al., DarkLight: a search for dark forces at the Jefferson laboratory free-electron laser facility, arXiv:1307.4432 [INSPIRE].

[55] WASA-AT-COSY collaboration, P. Adlarson et al., Search for a dark photon in the $\pi^{0} \rightarrow e^{+} e^{-} \gamma$ decay, Phys. Lett. B 726 (2013) 187 [arXiv:1304.0671] [InSPIRE].

[56] HADES collaboration, G. Agakishiev et al., Searching a dark photon with HADES, Phys. Lett. B 731 (2014) 265 [arXiv:1311.0216] [INSPIRE].

[57] J. Blümlein and J. Brunner, New exclusion limits on dark gauge forces from proton Bremsstrahlung in beam-dump data, Phys. Lett. B 731 (2014) 320 [arXiv:1311.3870] [INSPIRE].

[58] S. Andreas et al., Proposal for an experiment to search for light dark matter at the SPS, arXiv: 1312.3309 [INSPIRE].

[59] M. Battaglieri et al., The heavy photon search test detector, arXiv:1406.6115 [INSPIRE].

[60] H. Merkel et al., Search at the Mainz microtron for light massive gauge bosons relevant for the muon g-2 anomaly, Phys. Rev. Lett. 112 (2014) 221802 [arXiv:1404.5502] [INSPIRE].

[61] BABAR collaboration, J.P. Lees et al., Search for a dark photon in $e^{+} e^{-}$collisions at BaBar, Phys. Rev. Lett. 113 (2014) 201801 [arXiv:1406.2980] [INSPIRE].

[62] PHENIX collaboration, A. Adare et al., Search for dark photons from neutral meson decays in $p+p$ and $d+A u$ collisions at $\sqrt{s_{\mathrm{NN}}}=200 \mathrm{GeV}$, arXiv:1409.0851 [INSPIRE].

[63] D. Kazanas, R.N. Mohapatra, S. Nussinov, V.L. Teplitz and Y. Zhang, Supernova bounds on the dark photon using its electromagnetic decay, Nucl. Phys. B 890 (2015) 17 [arXiv: 1410.0221] [INSPIRE].

[64] B. Echenard, R. Essig and Y.-M. Zhong, Projections for dark photon searches at Mu3e, JHEP 01 (2015) 113 [arXiv:1411.1770] [InSPIRE].

[65] D. Gorbunov, A. Makarov and I. Timiryasov, Decaying light particles on board the SHiP (I): signal rate estimates for hidden photons, arXiv:1411.4007 [INSPIRE].

[66] E. Goudzovski, Search for the dark photon in $\pi^{0}$ decays by NA48/2 at CERN, in MesonNet workshop, LNF, Frascati Italy September 2014. 
[67] D. Curtin et al., Exotic decays of the 125 GeV Higgs boson, Phys. Rev. D 90 (2014) 075004 [arXiv:1312.4992] [INSPIRE].

[68] S. Gopalakrishna, S. Jung and J.D. Wells, Higgs boson decays to four fermions through an Abelian hidden sector, Phys. Rev. D 78 (2008) 055002 [arXiv:0801.3456] [INSPIRE].

[69] C.-F. Chang, E. Ma and T.-C. Yuan, Multilepton Higgs decays through the dark portal, JHEP 03 (2014) 054 [arXiv: 1308.6071] [InSPIRE].

[70] A. Falkowski and R. Vega-Morales, Exotic Higgs decays in the golden channel, JHEP 12 (2014) 037 [arXiv: 1405.1095] [inSPIRE].

[71] J.M. Cline, G. Dupuis, Z. Liu and W. Xue, The windows for kinetically mixed Z'-mediated dark matter and the galactic center gamma ray excess, JHEP 08 (2014) 131 [arXiv: 1405.7691] [INSPIRE].

[72] I. Hoenig, G. Samach and D. Tucker-Smith, Searching for dilepton resonances below the $Z$ mass at the LHC, Phys. Rev. D 90 (2014) 075016 [arXiv:1408.1075] [INSPIRE].

[73] Muon G-2 collaboration, G.W. Bennett et al., Final report of the muon E821 anomalous magnetic moment measurement at BNL, Phys. Rev. D 73 (2006) 072003 [hep-ex/0602035] [INSPIRE].

[74] M. Davier, A. Hoecker, B. Malaescu and Z. Zhang, Reevaluation of the hadronic contributions to the muon $g-2$ and to $\alpha\left(M_{Z}^{2}\right)$, Eur. Phys. J. C 71 (2011) 1515 [Erratum ibid. C 72 (2012) 1874] [arXiv: 1010.4180] [INSPIRE].

[75] N. Arkani-Hamed, D.P. Finkbeiner, T.R. Slatyer and N. Weiner, A theory of dark matter, Phys. Rev. D 79 (2009) 015014 [arXiv:0810.0713] [INSPIRE].

[76] M. Pospelov and A. Ritz, Astrophysical signatures of secluded dark matter, Phys. Lett. B 671 (2009) 391 [arXiv:0810.1502] [INSPIRE].

[77] D.P. Finkbeiner and N. Weiner, Exciting dark matter and the INTEGRAL/SPI $511 \mathrm{keV}$ signal, Phys. Rev. D 76 (2007) 083519 [astro-ph/0702587] [INSPIRE].

[78] P. Fayet, Light spin 1/2 or spin 0 dark matter particles, Phys. Rev. D 70 (2004) 023514 [hep-ph/0403226] [INSPIRE].

[79] N. Arkani-Hamed and N. Weiner, LHC signals for a superunified theory of dark matter, JHEP 12 (2008) 104 [arXiv:0810.0714] [INSPIRE].

[80] C. Cheung, J.T. Ruderman, L.-T. Wang and I. Yavin, Kinetic mixing as the origin of light dark scales, Phys. Rev. D 80 (2009) 035008 [arXiv:0902.3246] [INSPIRE].

[81] M. Baumgart, C. Cheung, J.T. Ruderman, L.-T. Wang and I. Yavin, Non-Abelian dark sectors and their collider signatures, JHEP 04 (2009) 014 [arXiv:0901.0283] [INSPIRE].

[82] D.E. Morrissey, D. Poland and K.M. Zurek, Abelian hidden sectors at a GeV, JHEP 07 (2009) 050 [arXiv: 0904.2567] [INSPIRE].

[83] D0 collaboration, V.M. Abazov et al., Search for NMSSM Higgs bosons in the $h \rightarrow a a \rightarrow \mu \mu \mu \mu, \mu \mu \tau \tau$ channels using pp collisions at $\sqrt{s}=1.96$ TeV, Phys. Rev. Lett. 103 (2009) 061801 [arXiv:0905.3381] [INSPIRE].

[84] CMS collaboration, Search for a non-standard-model Higgs boson decaying to a pair of new light bosons in four-muon final states, Phys. Lett. B 726 (2013) 564 [arXiv:1210.7619] [INSPIRE]. 
[85] CMS collaboration, Search for light resonances decaying into pairs of muons as a signal of new physics, JHEP 07 (2011) 098 [arXiv:1106.2375] [INSPIRE].

[86] CMS collaboration, Search for a non-standard-model Higgs boson decaying to a pair of new light bosons in four-muon final states, CMS-PAS-HIG-13-010, CERN, Geneva Switzerland (2013).

[87] R. Essig, J. Kaplan, P. Schuster and N. Toro, On the origin of light dark matter species, submitted to Phys. Rev. D (2010) [arXiv:1004.0691] [INSPIRE].

[88] S.A. Abel, M.D. Goodsell, J. Jaeckel, V.V. Khoze and A. Ringwald, Kinetic mixing of the photon with hidden $\mathrm{U}(1) s$ in string phenomenology, JHEP 07 (2008) 124

[arXiv:0803.1449] [INSPIRE].

[89] M. Goodsell, S. Ramos-Sanchez and A. Ringwald, Kinetic mixing of U(1)s in heterotic orbifolds, JHEP 01 (2012) 021 [arXiv: 1110.6901] [INSPIRE].

[90] M. Goodsell, J. Jaeckel, J. Redondo and A. Ringwald, Naturally light hidden photons in LARGE volume string compactifications, JHEP 11 (2009) 027 [arXiv:0909.0515] [INSPIRE].

[91] R. Schabinger and J.D. Wells, A minimal spontaneously broken hidden sector and its impact on Higgs boson physics at the Large Hadron Collider, Phys. Rev. D 72 (2005) 093007 [hep-ph/0509209] [INSPIRE].

[92] M.J. Strassler, Why unparticle models with mass gaps are examples of hidden valleys, arXiv:0801.0629 [INSPIRE].

[93] A. Martin and T.S. Roy, The gold-plated channel for supersymmetric Higgs via Higgsphilic $Z^{\prime}$, arXiv:1103.3504 [INSPIRE].

[94] J. Alwall et al., The automated computation of tree-level and next-to-leading order differential cross sections and their matching to parton shower simulations, JHEP $\mathbf{0 7}$ (2014) 079 [arXiv: 1405.0301] [INSPIRE].

[95] A. Alloul, N.D. Christensen, C. Degrande, C. Duhr and B. Fuks, FeynRules $2.0-a$ complete toolbox for tree-level phenomenology, Comput. Phys. Commun. 185 (2014) 2250 [arXiv: 1310.1921] [INSPIRE].

[96] M.J. Strassler, Possible effects of a hidden valley on supersymmetric phenomenology, hep-ph/0607160 [INSPIRE].

[97] A. Falkowski, J.T. Ruderman, T. Volansky and J. Zupan, Hidden Higgs decaying to lepton jets, JHEP 05 (2010) 077 [arXiv: 1002.2952] [INSPIRE].

[98] Y.F. Chan, M. Low, D.E. Morrissey and A.P. Spray, LHC signatures of a minimal supersymmetric hidden valley, JHEP 05 (2012) 155 [arXiv:1112.2705] [INSPIRE].

[99] E. Gabrielli, M. Heikinheimo, B. Mele and M. Raidal, Dark photons and resonant monophoton signatures in Higgs boson decays at the LHC, Phys. Rev. D 90 (2014) 055032 [arXiv:1405.5196] [INSPIRE].

[100] Particle Data Group collaboration, K. Olive et al., Review of particle physics (RPP), Chin. Phys. C 38 (2014) 090001 [INSPIRE].

[101] K.G. Chetyrkin, R.V. Harlander and J.H. Kuhn, Quartic mass corrections to $R_{\text {had }}$ at order $\alpha_{s}^{3}$, Nucl. Phys. B 586 (2000) 56 [Erratum ibid. B 634 (2002) 413] [hep-ph/0005139] [INSPIRE]. 
[102] K.G. Chetyrkin, J.H. Kuhn and A. Kwiatkowski, QCD corrections to the $e^{+} e^{-}$cross-section and the $Z$ boson decay rate: concepts and results, Phys. Rept. 277 (1996) 189 [INSPIRE].

[103] K.G. Chetyrkin, J.H. Kuhn and M. Steinhauser, RunDec: a Mathematica package for running and decoupling of the strong coupling and quark masses, Comput. Phys. Commun. 133 (2000) 43 [hep-ph/0004189] [INSPIRE].

[104] GFitTer Group collaboration, M. Baak et al., The global electroweak fit at NNLO and prospects for the LHC and ILC, Eur. Phys. J. C 74 (2014) 3046 [arXiv: 1407.3792] [INSPIRE].

[105] M. Baak et al., The electroweak fit of the standard model after the discovery of a new boson at the LHC, Eur. Phys. J. C 72 (2012) 2205 [arXiv:1209.2716] [InSPIRE].

[106] B. Batell, S. Gori and L.-T. Wang, Higgs couplings and precision electroweak data, JHEP 01 (2013) 139 [arXiv: 1209.6382] [inSPIRE].

[107] M. Baak et al., Working group report: precision study of electroweak interactions, arXiv:1310.6708 [INSPIRE].

[108] J.D. Wells and Z. Zhang, Precision electroweak analysis after the Higgs boson discovery, Phys. Rev. D 90 (2014) 033006 [arXiv:1406.6070] [INSPIRE].

[109] H. Flacher et al., Revisiting the global electroweak fit of the standard model and beyond with Gfitter, Eur. Phys. J. C 60 (2009) 543 [Erratum ibid. C 71 (2011) 1718] [arXiv:0811.0009] [INSPIRE].

[110] A. Falkowski and F. Riva, Model-independent precision constraints on dimension-6 operators, arXiv:1411.0669 [INSPIRE].

[111] M. Awramik, M. Czakon, A. Freitas and G. Weiglein, Precise prediction for the $W$ boson mass in the standard model, Phys. Rev. D 69 (2004) 053006 [hep-ph/0311148] [INSPIRE].

[112] G.-C. Cho, K. Hagiwara, Y. Matsumoto and D. Nomura, The MSSM confronts the precision electroweak data and the muon g-2, JHEP 11 (2011) 068 [arXiv:1104.1769] [INSPIRE].

[113] M. Awramik, M. Czakon and A. Freitas, Electroweak two-loop corrections to the effective weak mixing angle, JHEP 11 (2006) 048 [hep-ph/0608099] [INSPIRE].

[114] A. Freitas and Y.-C. Huang, Electroweak two-loop corrections to $\sin ^{2} \theta_{\text {eff }}^{b \bar{b}}$ and $R_{b}$ using numerical Mellin-Barnes integrals, JHEP 08 (2012) 050 [Erratum ibid. 05 (2013) 074] [Erratum ibid. 10 (2013) 044] [arXiv:1205.0299] [INSPIRE].

[115] A. Freitas, Higher-order electroweak corrections to the partial widths and branching ratios of the $Z$ boson, JHEP 04 (2014) 070 [arXiv:1401.2447] [INSPIRE].

[116] ALEPH, DELPHI, L3, OPAL, SLD, LEP Electroweak Working Group, SLD Electroweak Group and SLD Heavy Flavour Group collaborations, S. Schael et al., Precision electroweak measurements on the $Z$ resonance, Phys. Rept. 427 (2006) 257 [hep-ex/0509008] [INSPIRE].

[117] S. Moch et al., High precision fundamental constants at the TeV scale, arXiv:1405.4781 [INSPIRE].

[118] CMS collaboration, Projected improvement of the accuracy of top-quark mass measurements at the upgraded LHC, CMS-PAS-FTR-13-017, CERN, Geneva Switzerland (2013).

[119] S. Dawson et al., Working group report: Higgs boson, arXiv:1310.8361 [INSPIRE].

[120] J. Fan, M. Reece and L.-T. Wang, Possible futures of electroweak precision: ILC, FCC-ee and $C E P C$, arXiv:1411.1054 [INSPIRE]. 
[121] H. Baer et al., The International Linear Collider technical design report - volume 2: physics, arXiv:1306.6352 [INSPIRE].

[122] CMS collaboration, Properties of the Higgs-like boson in the decay $H \rightarrow Z Z \rightarrow 4 \ell$ in pp collisions at $\sqrt{s}=7$ and 8 TeV, CMS-PAS-HIG-13-002, CERN, Geneva Switzerland (2013).

[123] ATLAS collaboration, Measurements of the properties of the Higgs-like boson in the four lepton decay channel with the ATLAS detector using $25 \mathrm{fb}^{-1}$ of proton-proton collision data, ATLAS-CONF-2013-013, CERN, Geneva Switzerland (2013).

[124] CMS collaboration, Measurement of the properties of a Higgs boson in the four-lepton final state, Phys. Rev. D 89 (2014) 092007 [arXiv: 1312.5353] [INSPIRE].

[125] ATLAS collaboration, Measurements of the properties of the Higgs-like boson in the four lepton decay channel with the ATLAS detector using $25 \mathrm{fb}^{-1}$ of proton-proton collision data, ATLAS-CONF-2013-013, CERN, Geneva Switzerland (2013).

[126] CMS collaboration, Electron performance with $19.6 \mathrm{fb}^{-1}$ of data collected at $\sqrt{s}=8$ TeV with the CMS detector, CMS-DP-2013-003, CERN, Geneva Switzerland (2013).

[127] CMS collaboration, CMS tracking performance results from early LHC operation, Eur. Phys. J. C 70 (2010) 1165 [arXiv:1007.1988] [InSPIRE].

[128] J. Alwall, M. Herquet, F. Maltoni, O. Mattelaer and T. Stelzer, MadGraph 5: going beyond, JHEP 06 (2011) 128 [arXiv:1106.0522] [INSPIRE].

[129] T. Sjöstrand, S. Mrenna and P.Z. Skands, A brief introduction to PYTHIA 8.1, Comput. Phys. Commun. 178 (2008) 852 [arXiv:0710.3820] [INSPIRE].

[130] Higgs cross sections for HL-LHC and HE-LHC webpage, https://twiki.cern.ch/twiki/bin/view/LHCPhysics/HiggsEuropeanStrategy, (2014).

[131] LHC Higgs Cross section Working Group collaboration, S. Heinemeyer et al., Handbook of LHC Higgs cross sections: 3. Higgs properties, arXiv: 1307.1347 [INSPIRE].

[132] G. Bozzi, S. Catani, D. de Florian and M. Grazzini, Transverse-momentum resummation and the spectrum of the Higgs boson at the LHC, Nucl. Phys. B 737 (2006) 73 [hep-ph/0508068] [INSPIRE].

[133] D. de Florian, G. Ferrera, M. Grazzini and D. Tommasini, Transverse-momentum resummation: Higgs boson production at the Tevatron and the LHC, JHEP 11 (2011) 064 [arXiv: 1109.2109] [INSPIRE].

[134] J.M. Campbell, R.K. Ellis and C. Williams, Vector boson pair production at the LHC, JHEP 07 (2011) 018 [arXiv:1105.0020] [INSPIRE].

[135] ATLAS collaboration, Projections for measurements of Higgs boson cross sections, branching ratios and coupling parameters with the ATLAS detector at a HL-LHC, ATL-PHYS-PUB-2013-014, CERN, Geneva Switzerland (2013).

[136] CMS collaboration, Measurement of the differential and double-differential Drell-Yan cross sections in proton-proton collisions at $\sqrt{s}=7$ TeV, JHEP 12 (2013) 030 [arXiv: 1310.7291] [INSPIRE].

[137] ATLAS collaboration, Search for high-mass dilepton resonances in $20 \mathrm{fb}^{-1}$ of pp collisions at $\sqrt{s}=8 \mathrm{TeV}$ with the ATLAS experiment, ATLAS-CONF-2013-017, CERN, Geneva Switzerland (2013). 
[138] J. Jaeckel, M. Jankowiak and M. Spannowsky, LHC probes the hidden sector, Phys. Dark Univ. 2 (2013) 111 [arXiv:1212.3620] [INSPIRE].

[139] ATLAS collaboration, Measurement of the total $Z Z$ production cross section in proton-proton collisions at $\sqrt{s}=8 \mathrm{TeV}$ in $20 \mathrm{fb}^{-1}$ with the ATLAS detector, ATLAS-CONF-2013-020, CERN, Geneva Switzerland (2013).

[140] J.D. Clarke, R. Foot and R.R. Volkas, Phenomenology of a very light scalar $\left(100 \mathrm{MeV}<m_{h}<10 \mathrm{GeV}\right)$ mixing with the SM Higgs, JHEP 02 (2014) 123 [arXiv: 1310.8042] [INSPIRE].

[141] ATLAS collaboration, Search for long-lived neutral particles decaying into lepton jets in proton-proton collisions at $\sqrt{s}=8 \mathrm{TeV}$ with the ATLAS detector, JHEP 11 (2014) 088 [arXiv: 1409.0746] [INSPIRE].

[142] ATLAS collaboration, Search for invisible decays of a Higgs boson produced in association with a $Z$ boson in ATLAS, ATLAS-CONF-2013-011, CERN, Geneva Switzerland (2013).

[143] CMS collaboration, Search for invisible decays of Higgs bosons in the vector boson fusion and associated $Z H$ production modes, Eur. Phys. J. C 74 (2014) 2980 [arXiv:1404.1344] [INSPIRE].

[144] M.E. Peskin, Estimation of LHC and ILC capabilities for precision Higgs boson coupling measurements, arXiv:1312.4974 [INSPIRE].

[145] CMS collaboration, $H \rightarrow Z Z \rightarrow 4 \ell$, CMS-PAS-FTR-13-003, CERN, Geneva Switzerland (2013).

[146] G. Isidori, A.V. Manohar and M. Trott, Probing the nature of the Higgs-like boson via $h \rightarrow V \mathcal{F}$ decays, Phys. Lett. B 728 (2014) 131 [arXiv:1305.0663] [INSPIRE].

[147] A.L. Kagan et al., An exclusive window onto Higgs Yukawa couplings, arXiv:1406.1722 [INSPIRE].

[148] M. Gonzalez-Alonso and G. Isidori, The $h \rightarrow 4 \ell$ spectrum at low $m_{34}$ : standard model vs. light new physics, Phys. Lett. B 733 (2014) 359 [arXiv: 1403.2648] [INSPIRE].

[149] D.Y. Bardin et al., ZFITTER v.6.21: a semianalytical program for fermion pair production in $e^{+} e^{-}$annihilation, Comput. Phys. Commun. 133 (2001) 229 [hep-ph/9908433] [INSPIRE].

[150] ATLAS, CDF, CMS and D0 collaborations, First combination of Tevatron and LHC measurements of the top-quark mass, arXiv:1403.4427 [INSPIRE].

[151] CMS collaboration, Measurement of the top-quark mass in $t \bar{t}$ events with lepton+jets final states in pp collisions at $\sqrt{s}=8 \mathrm{TeV}$, CMS-PAS-TOP-14-001, CERN, Geneva Switzerland (2014). 